\title{
Crossover from Peierls distortion to one-dimensional superconductivity in arrays of $(5,0)$ carbon nanotubes
}

\author{
Ting Zhang, Ming Yuan Sun, Zhe Wang, Wu Shi, and Ping Sheng* \\ Department of Physics and William Mong Institute of Nano Science and Technology, Hong Kong University of Science and Technology, \\ Clear Water Bay, Kowloon, Hong Kong \\ (Received 11 May 2011; revised manuscript received 6 November 2011; published 29 December 2011)
}

\begin{abstract}
By performing second-order renormalization group analysis on thin arrays of 4-Angstrom $(5,0)$ carbon nanotubes (CNTs) embedded in aluminophosphate-five (AFI) zeolite crystals, we identify singlet superconductivity instability to be dominant at low temperatures, attributable to the screening of the electron-electron Coulomb interaction in the array configuration. Our analysis also shows that there is a crossover as the system scales to lower energy/temperatures, whereby one-dimensional (1D) superconductivity is the ground state, but the response function of the Peierls distortion/charge density wave (CDW) order dominates at the higher energy regime. This crossover behavior indicates that for a thin array of $(5,0) \mathrm{CNTs}$ the CDW order may represent an excited state of the array, so that the CDW characteristics can appear at finite temperatures, in conjunction with 1D superconductivity. Experimental results are presented to support this interpretation.
\end{abstract}

DOI: 10.1103/PhysRevB.84.245449 PACS number(s): 74.20.-z, 73.63.Fg, 74.25.F-, 74.70.Wz

\section{INTRODUCTION}

The electronic ground state of single-wall carbon nanotubes (SWCNTs) is an interesting and still unresolved problem. Owing to the variety of SWCNT structures, their ground state may not be unique. This can be true even for the metallic SWCNTs. Besides the well-known Luttinger liquid (LL) state, intrinsic superconductivity has been experimentally observed or theoretically predicted in SWCNTs,${ }^{1-13}$ CNT ropes, ${ }^{14-17}$ multiwall CNTs (MWCNTs), ${ }^{18-23}$ and doped CNTs. ${ }^{24-27}$ For the case of ultrathin diameter 4-Angstrom metallic SWCNTs, experimental observation of the Meissner effect in $2001^{1}$ has prompted some theoretical works exploring the possibility of superconductivity being the ground state of the system. While some authors predicted Peierls ground state, ${ }^{28-32}$ others predicted superconductivity. ${ }^{33-40}$ More recently, the observation of the superconducting electrical transition for arrays of aligned 4-Angstrom nanotubes (embedded in AFI zeolite crystals) has shown that the resistance drop is precipitated by a BerezinskiiKosterlitz-Thouless (BKT)-like transition ${ }^{41-43}$ in the plane perpendicular to the $c$-axis of the nanotubes, thereby facilitating a $1 \mathrm{D}$ to $3 \mathrm{D}$ crossover that leads to bulk superconductivity ${ }^{2-4}$ at low temperatures. Three dimensional magnetic Meissner characteristics was subsequently also observed on this low temperature state. ${ }^{5}$ Such experimental evidences thus strongly suggest the important role of transverse coupling between the SWCNTs in determining the ground state.

In this work we study the ground state of thin arrays of $(5,0)$ 4-Angstrom CNTs. The $(5,0)$ CNTs are noted to have three bands crossing the Fermi level, denoted as A, $\mathrm{A}^{\prime}$, and B. This feature implies that it cannot be completely similar to the LL $^{44-51}$ behavior. Theoretically, the LL model may break down in ultrathin CNTs because in the presence of phonon-mediate electron-electron interactions, the system may deviate from the Luttinger fixed point if the net effective interaction is attractive, which would imply scaling to the strong coupling limit. ${ }^{52-58}$ Some earlier works ${ }^{59-61}$ have analyzed the ultrathin SWCNT system with bosonization ${ }^{46-49}$ and first-order renormalization group (RG) approaches. ${ }^{52-58}$ However, whereas bosoniztion suffers from the perturbative nature in treating the backward scattering, first-order RG can lead to unphysical divergence in the attractive coupling regime.

Here we report the results of the second-order $\mathrm{RG}^{52-58}$ analysis in calculating the electronic instabilities of thin arrays of $(5,0)$ CNTs. The differential resistance behavior of such thin arrays is predicted by using the Landauer formula. It is shown that for a single $(5,0)$ carbon nanotube $(\mathrm{CNT})$, the ground state at low temperatures is governed by the Peierls distortion, or the charge density wave (CDW) state, unless the $(5,0) \mathrm{CNT}$ is embedded in a very high dielectric constant matrix. But for a thin array of $(5,0) \mathrm{CNTs}$ embedded in the AFI crystal (with a dielectric constant $\sim 6$ ), comprising one central $(5,0)$ CNT surrounded by six others with an array cross-sectional diameter of $\sim 3 \mathrm{~nm}$, there can be a Peierls-1D superconductivity crossover as the temperature is lowered, with the superconducting state emerging as the ground state. We interpret this to mean that the CDW state can be an excited state of the thin $(5,0)$ array so that at finite temperatures the characteristics of the Peierls/CDW order can appear even in the context of a 1D superconducting ground state, and this interpretation can provide a qualitative explanation to the otherwise puzzling differential resistance observations on some selective samples.

In what follows Sec. II is devoted to a RG treatment of the system. Description of the formalism in Sec. II A is followed by a presentation of the relevant methodology in Sec. II B. In Sec. II C we consider the fixed points of the renormalization as obtained by integrating from the physically reasonable initial values. In Sec. III we present the device characteristics for the Peierls/CDW order by considering the effect of the electrical leads. Based on the results obtained, in Sec. IV we give an interpretation of the differential resistance behavior observed in some selective samples of the 4-Angstrom CNTs' system.

\section{RG ANALYSIS OF THE COUPLING CONSTANTS AND THE RESPONSE FUNCTIONS}

\section{A. Interaction Hamiltonian, channels, and coupling constants}

The Hamiltonian of a thin array of $(5,0)$ CNTs, comprising a central CNT surrounded by six others, can be expressed as 

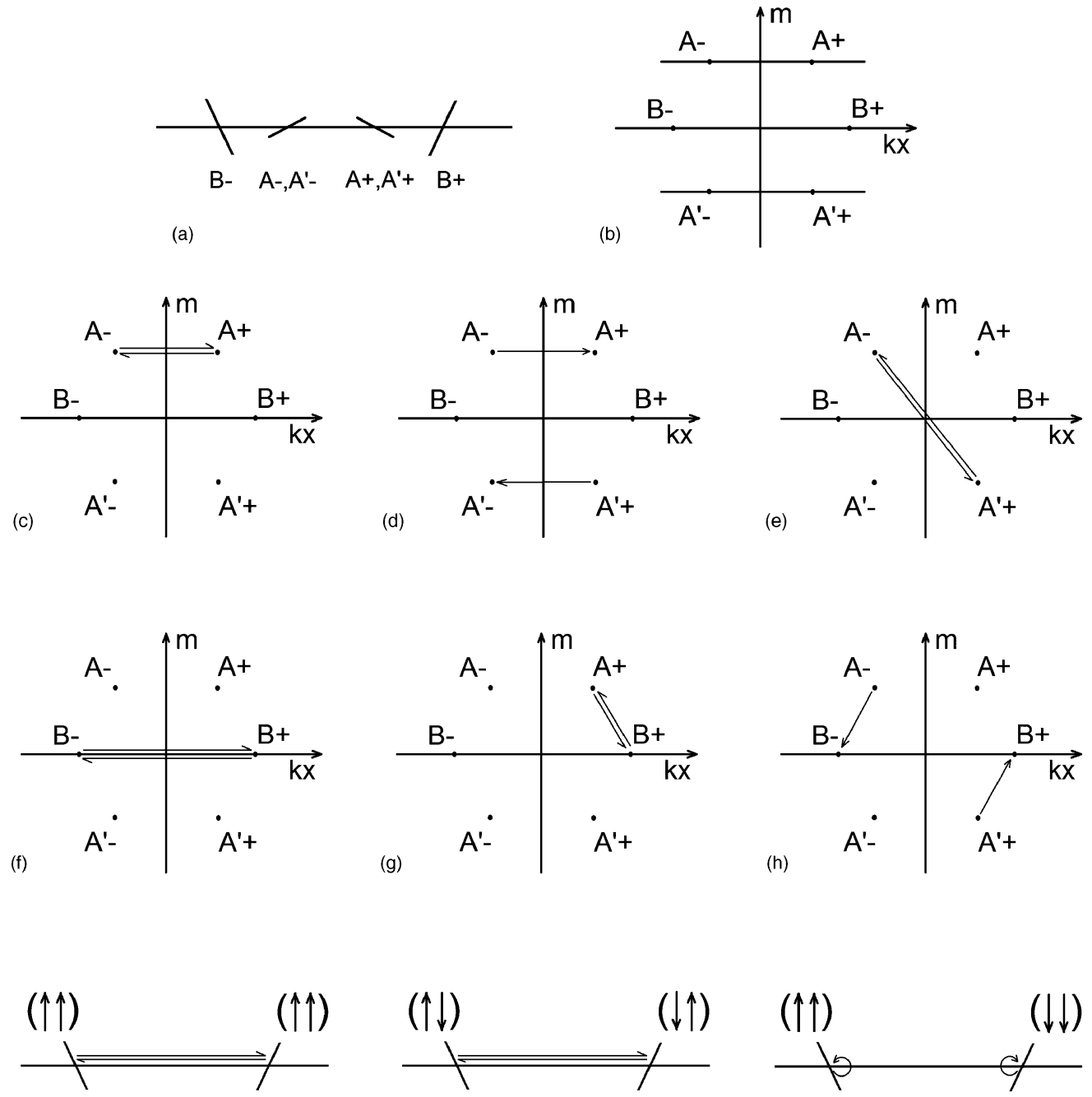

(i)

(j)

(k)

FIG. 1. (a) The linearized single electron-band structure near Fermi level. A and A' bands are degenerate, with opposite Fermi velocity to that in the $\mathrm{B}$ band. (b) The Brillouin zone. The horizontal axis is $k$ along the tube axis, while the vertical axis is angular momentum around the tube circumference. The black dots denoted by $\mathrm{A} \pm, \mathrm{B} \pm$ are the Fermi points in the $1 \mathrm{D}$ band. (c)-(h) show the schematic diagrams for the six intratube channels. (i)-(k) illustrate $g_{1 \|}, g_{1 \perp}$, and $g_{2}$ in each channel.

the sum of a linearized noninteracting part plus a part containing the electron-electron interaction:

$$
\begin{aligned}
H= & \sum_{\sigma=\uparrow, \downarrow} \sum_{m} \sum_{k}\left(\varepsilon_{k_{F} m} a_{k m \sigma}^{+} a_{k m \sigma}+\varepsilon_{-k_{F} m} b_{k m \sigma}^{+} b_{k m \sigma}\right)+\sum_{\sigma, \sigma^{\prime}} \sum_{n} \sum_{k, p, q}\left(g_{n}^{11} a_{k n \sigma}^{+} b_{p n \sigma^{\prime}}^{+} a_{p+2 k_{F}+q, n, \sigma^{\prime}} b_{k-2 k_{F}-q, n, \sigma} \delta_{\sigma, \sigma^{\prime}}\right. \\
& \left.+g_{n}^{1 \perp} a_{k n \sigma}^{+} b_{p n \sigma^{\prime}}^{+} a_{p+2 k_{F}+q, n, \sigma^{\prime}} b_{k-2 k_{F}-q, n, \sigma} \delta_{\sigma,-\sigma^{\prime}}+g_{n}^{2} a_{k \sigma}^{+} b_{p, \sigma^{\prime}}^{+} b_{p+q, \sigma^{\prime}} a_{k-q, \sigma} \delta_{\sigma,-\sigma^{\prime}}\right),
\end{aligned}
$$

where $k, p$, and $q$ denote momentum, $a$ and $b$ are annihilation operators of electrons for $k$ values around the Fermi momentum in the electron bands $k_{F m}$ and $-k_{F m}$, respectively. Index $m$ denotes the three partially fulfilled electron bands in the $(5,0)$ band structure. Here $m=0$ stands for the B band and $m= \pm 1$ stands, respectively, for the $\mathrm{A}$ and $\mathrm{A}^{\prime}$ bands, as shown in Figs. 1(a) and 1(b). Index $\sigma$ means spin, and $\varepsilon_{k_{F} m}$ denotes the energy dispersion around the Fermi point of band $m$. In the interaction part of the Hamiltonian, $n$ denotes the different electron-electron interaction channels, shown in Figs. 1(c)-1(h), in which $n=1$ means the channel shown in Fig. 1(c), $n=2$ the channels in Fig. 1(d), etc. Here a channel is defined as comprising a left-propagating electron together with a right-propagating electron. For later considerations it is noted that only if the two electrons are such that one is left-moving and the other one rightmoving, can there appear logarithmically divergent expansion diagrams. 
The two electrons interact with each other with a coupling constant, which can differ from channel to channel. Within each channel there can be three types of interactions. These three are schematically illustrated in Figs. 1(i)-1(k), in which the coupling constant $g_{1 \|} / g_{1 \perp}$ is relevant to backward scattering with spins parallel/antiparallel, while $g_{2}$ denotes forward scattering. Hence there are in total 18 intratube coupling constants. If we consider intertube interactions within a thin array, consisting of a central nanotube surrounded by six others, one only needs to consider a pair of the nearest neighbors, and there can be 12 different channels. Hence there can be 36 intertube coupling constants, and these couplings should be taken into account six times, one for each surrounding CNT. As will be seen subsequently, the coupling constants are the ones to be renormalized in the RG analysis.

\section{B. Derivation of scaling equations for the coupling constants}

We follow the formalism presented in the literature ${ }^{52-58}$ to perform the RG analysis up to the second order. The scaling equation of coupling constants $g_{i}$ versus cutoff energy $E_{0}$ can be generally expressed as

$$
\begin{aligned}
g_{n}^{j \prime}= & g_{n}^{j}\left\{\left(d_{m}\left(\left(E_{0}^{\prime} / E_{0}\right), g\right) d_{m^{\prime}}\left(\left(E_{0}^{\prime} / E_{0}\right), g\right)\right) /\left(d_{m}\left(1, g^{\prime}\right)\right.\right. \\
& \left.\left.\times d_{m^{\prime}}\left(1, g^{\prime}\right)\right)\right\}\left\{\widetilde{\Gamma}_{n, j}\left[\left(E_{0}^{\prime} / E_{0}\right), g\right] / \widetilde{\Gamma}_{n, j}\left(1, g^{\prime}\right)\right\} .
\end{aligned}
$$

Here $g^{\prime}$ is the new (renormalized) coupling constant and $g$ is the original coupling constant; $n$ is the channel index; $j$ denotes $1 / /, 1 \perp$ or $2 ; \widetilde{\Gamma}_{n}^{j}=H_{\text {ele-ele }, n}^{j} / g_{n}^{j}$ is the dimensionless interaction vertex (where $H_{\text {ele-ele }}$ just denotes the second part on the right-hand side of Eq. (1)); and $d_{m\left(m^{\prime}\right)}=G_{m\left(m^{\prime}\right)} / G_{m\left(m^{\prime}\right)}{ }^{(0)}$ is the dimensionless Green function in band $m\left(m^{\prime}\right)$ associated within each channel $n$. The dimensionless interaction vertex and Green function can be perturbatively expanded with the Feynman diagram technique. The integral over intermediate states would then yield terms that contain $\ln \left(\omega / E_{0}\right)$, which are divergent in the low energy limit. The RG technique is

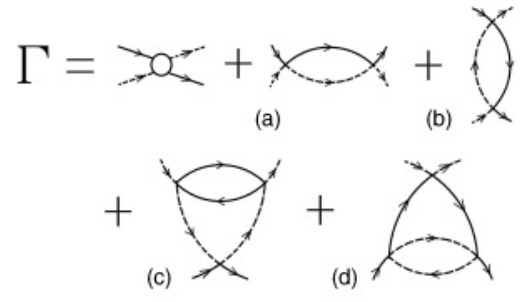

FIG. 2. The logarithmically divergent diagrams, (a), (b), (c), and (d), in the perturbative expansion of the interaction vertex associated with the second-order RG. Nondivergent diagrams and higher-order diagrams are not shown. Here a solid line denotes the right-moving electron, while a dashed line denotes the left-moving electron.

to reduce the cutoff $E_{0}$ to a smaller $E_{0}{ }^{\prime}$ and to integrate the states between $E_{0}{ }^{\prime}$ and $E_{0}$. A smaller cutoff will cause the logarithmic function to be less divergent. In this process the coupling constants must be changed a bit to maintain the overall physical characteristic, and this change is described by Eq. (2), denoted the scaling equation, which the electronelectron couplings must obey.

The electron-electron interaction vertex can be perturbatively expanded by using the Feynman diagram technique up to the two-loop cases, shown in Fig. 2. All vertices must satisfy the momentum and energy conservation laws, and inner lines run over all possible states. We integrate inner lines over their energy and momenta. These middle states are expressed in free-particle Green function as

$$
G_{m \pm}(k, \omega)=\frac{1}{\omega-\varepsilon_{m \pm}(k)+i \delta \operatorname{sgn}\left[\varepsilon_{m \pm}(k)\right]},
$$

where $\varepsilon_{m \pm}(k)=v_{F m}\left(k-k_{F m \pm}\right)$ is the energy dispersion, $i \delta$ is an infinitesimal imaginary constant, and sgn denotes the function of taking the sign of the argument. The diagrams shown in Figs. 2(a) and 2(b) are called Bardeen-Cooper-Shrieffer (BCS) and zero-sound diagrams, respectively. Their integrals are singular if two inner lines have opposite group velocities, i.e.,

$$
\begin{gathered}
-i \int \frac{d k^{\prime}}{2 \pi} \int \frac{d \omega^{\prime}}{2 \pi} G_{m, \text { right-moving }}\left(k^{\prime}, \omega^{\prime}\right) G_{m^{\prime}, \text { left-moving }}\left(-k^{\prime}, \omega-\omega^{\prime}\right)=-\frac{1}{\pi\left(v_{F m}+v_{F m^{\prime}}\right)} \ln \left(\frac{\omega}{\pi\left(v_{F m}+v_{F m^{\prime}}\right) k_{C}}\right) \\
-i \int \frac{d k^{\prime}}{2 \pi} \int \frac{d \omega^{\prime}}{2 \pi} G_{m, \text { right-moving }}\left(k^{\prime}, \omega^{\prime}\right) G_{m^{\prime}, \text { left-moving }}\left(k^{\prime}-\Delta k, \omega^{\prime}-\omega\right)=\frac{1}{\pi\left(v_{F m}+v_{F m^{\prime}}\right)} \ln \left(\frac{\omega}{\pi\left(v_{F m}+v_{F m^{\prime}}\right) k_{C}}\right),
\end{gathered}
$$

in which $k_{C}$ denotes momentum cutoff and $\Delta k$ denotes difference in momenta between two associated Fermi points. Here all group velocities are taken to be their absolute value.

In general these diagrams give rise to terms in the form of $g^{2} \ln \left(\omega / E_{0}\right)$, and by collecting all these terms we can derive the RG-scaling functions up to the first order. The next corrections to the scaling equations come from Figs. 2(c) and 2(d), which give rise to terms in form of $g^{3} \ln \left(\omega / E_{0}\right)$. By collecting diagrams like those shown in Figs. 2(c) and 2(d), we generate the second-order scaling equations. Here in Fig. 3 we give an example for all the diagrams for $\Gamma_{1}^{2}$, which belongs to the $g_{2}$-type interaction vertex in the A band.

To be consistent, we must also take into account the second-order self-energy correction to the free-particle Green function, shown in Fig. 4. By following Ref. 56, the corrections to the Green function and the group velocities are given by

$$
\begin{aligned}
G_{A-}(k, \omega)= & \frac{1}{\omega-v_{A}^{\prime} k}\left[1+\sum_{n \neq A A^{\prime} B B} \frac{1}{2 \pi^{2}\left(v_{m}^{\prime}+v_{m^{\prime}}^{\prime}\right)^{2}}\left(\left(g_{n}^{1 \|}-g_{n}^{2}\right)^{2}+\left(g_{n}^{1 \perp}\right)^{2}+\left(g_{n}^{2}\right)^{2}\right) \ln \left(\omega / E_{0}\right)\right. \\
& \left.+\frac{1}{4 \pi^{2} v_{B}^{\prime}\left(v_{A}^{\prime}+v_{B}^{\prime}\right)}\left(\left(g_{A A^{\prime} B B}^{1 \|}-g_{A A^{\prime} B B}^{2}\right)^{2}+\left(g_{A A^{\prime} B B}^{1 \perp}\right)^{2}+\left(g_{A A^{\prime} B B}^{2}\right)^{2}\right) \ln \left(\omega / E_{0}\right)\right]
\end{aligned}
$$



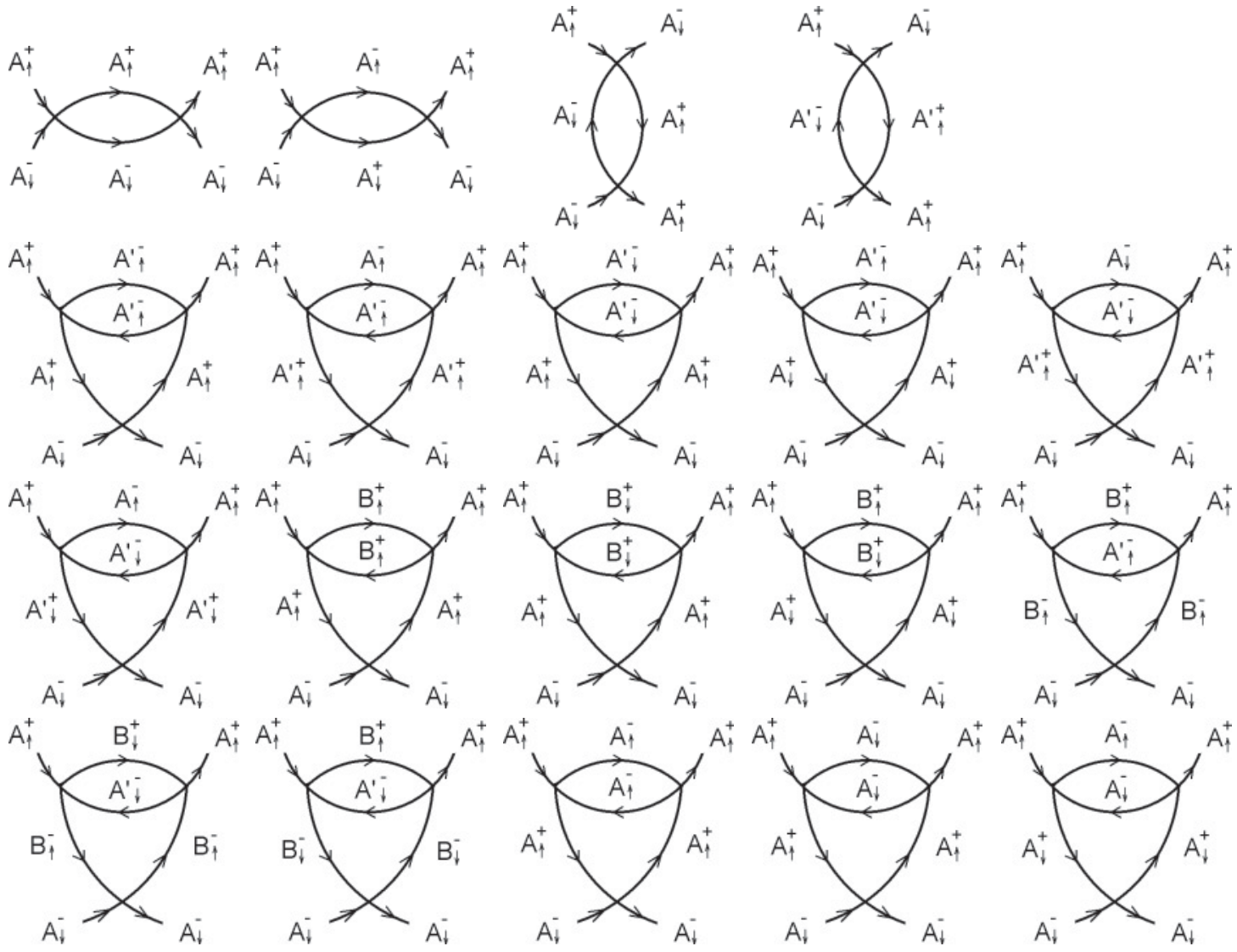

FIG. 3. The diagrams that give rise to $\ln \left(\omega / E_{0}\right)$ for $\Gamma_{1}^{2}$. The first four diagrams belong to the first-order RG diagrams, while others belong to the second-order RG diagrams. The down-triangle diagrams are similar to the up-triangle ones and hence not listed.

$$
\begin{aligned}
G_{B+}(k, \omega)= & \frac{1}{\omega-v_{B}^{\prime} k}\left[1+\sum_{n \neq A A^{\prime} B B} \frac{1}{2 \pi^{2}\left(v_{m}^{\prime}+v_{m^{\prime}}^{\prime}\right)^{2}}\left(\left(g_{n}^{1 \|}-g_{n}^{2}\right)^{2}+\left(g_{n}^{1 \perp}\right)^{2}+\left(g_{n}^{2}\right)^{2}\right) \ln \left(\omega / E_{0}\right)\right. \\
& \left.+\frac{2}{4 \pi^{2} v_{A}^{\prime}\left(v_{A}^{\prime}+v_{B}^{\prime}\right)}\left(\left(g_{A A^{\prime} B B}^{1 \|}-g_{A A^{\prime} B B}^{2}\right)^{2}+\left(g_{A A^{\prime} B B}^{1 \perp}\right)^{2}+\left(g_{A A^{\prime} B B}^{2}\right)^{2}\right) \ln \left(\omega / E_{0}\right)\right] \\
v_{A}^{\prime}= & v_{A}+\left(v_{A}-v_{B}\right) \frac{v_{A}+v_{B}}{2 v_{B}}\left(\left(g_{A A^{\prime} B B}^{1 \|}-g_{A A^{\prime} B B}^{2}\right)^{2}+\left(g_{A A^{\prime} B B}^{1 \perp}\right)^{2}+\left(g_{A A^{\prime} B B}^{2}\right)^{2}\right) \ln \left(\omega / E_{0}\right) \\
v_{B}^{\prime}= & v_{B}+\left(v_{B}-v_{A}\right) \frac{v_{A}+v_{B}}{2 v_{A}}\left(\left(g_{A A^{\prime} B B}^{1 \|}-g_{A A^{\prime} B B}^{2}\right)^{2}+\left(g_{A A^{\prime} B B}^{1 \perp}\right)^{2}+\left(g_{A A^{\prime} B B}^{2}\right)^{2}\right) \ln \left(\omega / E_{0}\right),
\end{aligned}
$$
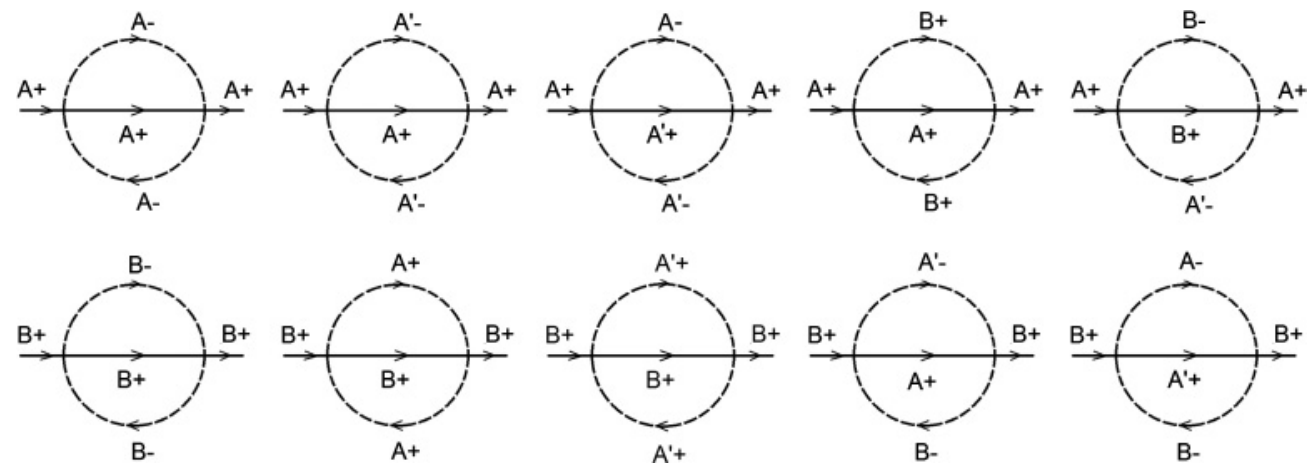

FIG. 4. The diagrams for the second-order self-energy correction to the Green function. The first five diagrams (top row) are related to the $\mathrm{A} / \mathrm{A}^{\prime}$ band, while the last five diagrams (bottom row) are related to the $\mathrm{B}$ band. 
where we can see that the difference between $v_{A}$ and $v_{B}$ vanishes with scaling and is therefore unimportant. Therefore we do not take the differnece between $v_{A}$ and $v_{B}$ into account in what follows.

From Eq. (5) we obtain the second-order dimensionless Green function, which can be combined with the vertex corrections shown in Figs. 2 and 3 to lead to the second-order RG equations using Eq. (2). Below we show the RG equations in the subspace of intratube couplings. The whole set of scaling equations containing intertube couplings are too tedious to list explicitly.

$$
\begin{aligned}
& \frac{d g_{1}^{1 \perp}}{d \ln \left(\omega / E_{0}\right)}=\frac{1}{2 \pi v_{A}}\left(-2 g_{2}^{1 \perp} g_{2}^{2}+2 g_{1}^{1 \perp} g_{1}^{1 \|}+2 g_{2}^{1 \perp} g_{2}^{1 \|}\right)+\frac{1}{\left(2 \pi v_{A}\right)^{2}}\left(g _ { 1 } ^ { 1 \perp } \left(\left(g_{1}^{1 \perp}\right)^{2}+\left(g_{1}^{1 \|}\right)^{2}+\left(g_{2}^{1 \perp}\right)^{2}\right.\right. \\
& \left.\left.+\left(g_{2}^{1 \|}\right)^{2}+\left(g_{3}^{1 \perp}\right)^{2}+\left(g_{3}^{1 \|}\right)^{2}\right)+2 g_{2}^{2}\left(g_{3}^{1 \perp}-g_{1}^{1 \perp}\right)\left(g_{2}^{1 \|}-g_{2}^{2}\right)\right)+\frac{1}{\pi^{2}\left(v_{A}+v_{B}\right)^{2}} g_{1}^{1 \perp}\left(\left(g_{5}^{1 \perp}\right)^{2}+\left(g_{5}^{1 \|}\right)^{2}\right) \\
& +\frac{1}{2 \pi^{2} v_{B}\left(v_{A}+v_{B}\right)}\left(2 g_{5}^{1 \perp} g_{6}^{2}\left(g_{6}^{1 \|}-g_{6}^{2}\right)+g_{1}^{1 \perp}\left(\left(g_{6}^{1 \|}-g_{6}^{2}\right)^{2}+\left(g_{6}^{1 \perp}\right)^{2}+\left(g_{6}^{2}\right)^{2}\right)\right) \\
& \frac{d g_{1}^{2}}{d \ln \left(\omega / E_{0}\right)}=\frac{1}{2 \pi v_{A}}\left(\left(g_{1}^{1 \perp}\right)^{2}-\left(g_{2}^{2}\right)^{2}\right) \\
& +\frac{1}{\left(2 \pi v_{A}\right)^{2}}\left(g_{1}^{1 \|}\left(\left(g_{1}^{1 \perp}\right)^{2}+\left(g_{3}^{1 \perp}\right)^{2}\right)+g_{3}^{1 \|}\left(g_{2}^{1 \perp}\right)^{2}-\left(g_{3}^{2}-g_{1}^{2}\right)\left(\left(g_{2}^{1 \|}-g_{2}^{2}\right)^{2}+\left(g_{2}^{1 \perp}\right)^{2}+\left(g_{2}^{2}\right)^{2}\right)\right) \\
& +\frac{1}{\pi^{2}\left(v_{A}+v_{B}\right)^{2}} g_{1}^{1 \|}\left(g_{5}^{1 \perp}\right)^{2}+\frac{1}{2 \pi^{2} v_{B}\left(v_{A}+v_{B}\right)}\left(g_{5}^{1 \|}\left(g_{6}^{1 \perp}\right)^{2}+\left(g_{1}^{2}-g_{5}^{2}\right)\left(\left(g_{6}^{1 \|}-g_{6}^{2}\right)^{2}+\left(g_{6}^{2}\right)^{2}+\left(g_{6}^{1 \perp}\right)^{2}\right)\right) \\
& \frac{d g_{1}^{1 \|}}{d \ln \left(\omega / E_{0}\right)}=\frac{1}{2 \pi v_{A}}\left(-2 g_{2}^{2} g_{2}^{1 \|}+2\left(g_{1}^{1 \perp}\right)^{2}+\left(g_{2}^{1 \perp}\right)^{2}+\left(g_{2}^{1 \|}\right)^{2}\right) \\
& +\frac{1}{\left(2 \pi v_{A}\right)^{2}}\left(2 g_{1}^{1 \|}\left(\left(g_{1}^{1 \perp}\right)^{2}+\left(g_{3}^{1 \perp}\right)^{2}\right)-\left(g_{3}^{1 \|}-g_{1}^{1 \|}\right)\left(\left(g_{2}^{1 \|}-g_{2}^{2}\right)^{2}+\left(g_{2}^{2}\right)^{2}-\left(g_{2}^{1 \perp}\right)^{2}\right)\right) \\
& +\frac{1}{\pi^{2}\left(v_{A}+v_{B}\right)^{2}}\left(2 g_{1}^{1 \|}\left(g_{5}^{1 \perp}\right)^{2}\right)+\frac{1}{2 \pi^{2} v_{B}\left(v_{A}+v_{B}\right)}\left(\left(g_{1}^{1 \|}-g_{5}^{1 \|}\right)\left(\left(g_{6}^{1 \|}-g_{6}^{2}\right)^{2}+\left(g_{6}^{2}\right)^{2}\right)+\left(g_{1}^{1 \|}+g_{5}^{1 \|}\right)\left(g_{6}^{1 \perp}\right)^{2}\right) \\
& \frac{d g_{2}^{1 \perp}}{d \ln \left(\omega / E_{0}\right)}=\frac{1}{2 \pi v_{B}}\left(2 g_{6}^{2} g_{6}^{1 \perp}\right)+\frac{1}{2 \pi v_{A}}\left(2 g_{3}^{1 \perp} g_{2}^{2}+2 g_{2}^{1 \perp}\left(g_{3}^{2}+g_{1}^{1 \|}-g_{1}^{2}\right)+2 g_{1}^{1 \perp}\left(g_{2}^{1 \|}-g_{2}^{2}\right)\right) \\
& +\frac{1}{\left(2 \pi v_{A}\right)^{2}} g_{2}^{1 \perp}\left(2 g_{3}^{2}\left(g_{1}^{1 \|}-g_{1}^{2}\right)+\left(g_{1}^{1 \|}-g_{1}^{2}\right)^{2}+2 g_{1}^{2}\left(g_{3}^{1 \|}-g_{3}^{2}\right)+\left(g_{3}^{1 \|}-g_{3}^{2}\right)^{2}+\left(g_{2}^{1 \|}-g_{2}^{2}\right)^{2}\right. \\
& \left.+\left(g_{2}^{2}\right)^{2}+\left(g_{2}^{1 \perp}\right)^{2}+\left(g_{3}^{2}\right)^{2}+\left(g_{3}^{1 \perp}\right)^{2}+\left(g_{1}^{2}\right)^{2}+\left(g_{1}^{1 \perp}\right)^{2}\right) \\
& +\frac{1}{\pi^{2}\left(v_{A}+v_{B}\right)^{2}} g_{2}^{1 \perp}\left(\left(g_{5}^{1 \|}\right)^{2}+\left(g_{5}^{1 \perp}\right)^{2}\right)+\frac{1}{2 \pi^{2} v_{B}\left(v_{A}+v_{B}\right)} g_{2}^{1 \perp}\left(\left(g_{6}^{1 \|}-g_{6}^{2}\right)^{2}+\left(g_{6}^{2}\right)^{2}+\left(g_{6}^{1 \perp}\right)^{2}\right) \\
& \frac{d g_{2}^{2}}{d \ln \left(\omega / E_{0}\right)}=\frac{1}{2 \pi v_{A}}\left(2 g_{3}^{1 \perp} g_{2}^{1 \perp}+2 g_{2}^{2}\left(g_{3}^{2}-g_{1}^{2}\right)\right)+\frac{1}{2 \pi v_{B}}\left(\left(g_{6}^{2}\right)^{2}+\left(g_{6}^{1 \perp}\right)^{2}\right)+\frac{1}{\left(2 \pi v_{A}\right)^{2}} 2 g_{1}^{1 \perp} g_{3}^{1 \perp}\left(g_{2}^{1 \|}-g_{2}^{2}\right) \\
& +\frac{1}{\pi^{2}\left(v_{A}+v_{B}\right)^{2}} g_{2}^{1 \|}\left(g_{5}^{1 \perp}\right)^{2}+\frac{1}{2 \pi^{2} v_{B}\left(v_{A}+v_{B}\right)} g_{2}^{2}\left(\left(g_{6}^{1 \|}-g_{6}^{2}\right)^{2}+\left(g_{6}^{2}\right)^{2}+\left(g_{6}^{1 \perp}\right)^{2}\right) \\
& +\frac{1}{\left(2 \pi v_{A}\right)^{2}} g_{2}^{2}\left(-2\left(g_{1}^{1 \|}-g_{1}^{2}\right)\left(g_{3}^{1 \|}-g_{3}^{2}\right)-2 g_{1}^{2} g_{3}^{2}+\left(g_{2}^{1 \|}-g_{2}^{2}\right)^{2}+\left(g_{2}^{2}\right)^{2}+\left(g_{2}^{1 \perp}\right)^{2}\right. \\
& \left.+\left(g_{3}^{1 \|}-g_{3}^{2}\right)^{2}+\left(g_{3}^{2}\right)^{2}+\left(g_{3}^{1 \perp}\right)^{2}+\left(g_{1}^{1 \|}-g_{1}^{2}\right)^{2}+\left(g_{1}^{2}\right)^{2}+\left(g_{1}^{1 \perp}\right)^{2}\right) \\
& \frac{d g_{2}^{1 \|}}{d \ln \left(\omega / E_{0}\right)}=\frac{1}{2 \pi v_{A}} 2\left(\left(g_{3}^{1 \|}-g_{1}^{1 \|}\right) g_{2}^{2}+\left(g_{1}^{1 \|}-g_{3}^{1 \|}+g_{3}^{2}-g_{1}^{2}\right) g_{2}^{1 \|}+\left(g_{1}^{1 \perp}+g_{3}^{1 \perp}\right) g_{2}^{1 \perp}\right) \\
& +\frac{1}{2 \pi v_{B}}\left(2 g_{6}^{2} g_{6}^{1 \|}-\left(g_{6}^{1 \|}\right)^{2}+\left(g_{6}^{1 \perp}\right)^{2}\right)+\frac{1}{\pi^{2}\left(v_{A}+v_{B}\right)^{2}} 2 g_{2}^{1 \|}\left(g_{5}^{1 \perp}\right)^{2} \\
& +\frac{1}{\left(2 \pi v_{A}\right)^{2}} g_{2}^{1 \|}\left(-2\left(g_{1}^{1 \|}-g_{1}^{2}\right)\left(g_{3}^{1 \|}-g_{3}^{2}\right)-2 g_{1}^{2} g_{3}^{2}+2 g_{1}^{1 \perp} g_{3}^{1 \perp}+\left(g_{2}^{1 \|}-g_{2}^{2}\right)^{2}+\left(g_{2}^{2}\right)^{2}\right. \\
& \left.+\left(g_{2}^{1 \perp}\right)^{2}+\left(g_{3}^{1 \|}-g_{3}^{2}\right)^{2}+\left(g_{3}^{2}\right)^{2}+\left(g_{3}^{1 \perp}\right)^{2}+\left(g_{1}^{1 \|}-g_{1}^{2}\right)^{2}+\left(g_{1}^{2}\right)^{2}+\left(g_{1}^{1 \perp}\right)^{2}\right) \\
& +\frac{1}{2 \pi^{2} v_{B}\left(v_{A}+v_{B}\right)} g_{2}^{1 \|}\left(\left(g_{6}^{1 \|}-g_{6}^{2}\right)^{2}+\left(g_{6}^{2}\right)^{2}+\left(g_{6}^{1 \perp}\right)^{2}\right)
\end{aligned}
$$




$$
\begin{aligned}
\frac{d g_{3}^{1 \perp}}{d \ln \left(\omega / E_{0}\right)}= & \frac{1}{2 \pi v_{A}}\left(2 g_{2}^{2} g_{2}^{1 \perp}+2 g_{3}^{1 \perp} g_{3}^{1 \|}\right)+\frac{1}{2 \pi v_{B}}\left(2 g_{6}^{2} g_{6}^{1 \perp}\right)+\frac{1}{\pi^{2}\left(v_{A}+v_{B}\right)^{2}} g_{3}^{1 \perp}\left(\left(g_{5}^{1 \|}\right)^{2}+\left(g_{5}^{1 \perp}\right)^{2}\right) \\
& +\frac{1}{2 \pi^{2} v_{B}\left(v_{A}+v_{B}\right)}\left(2 g_{5}^{1 \perp} g_{6}^{2}\left(g_{6}^{1 \|}-g_{6}^{2}\right)+g_{3}^{1 \perp}\left(\left(g_{6}^{1 \|}-g_{6}^{2}\right)^{2}+\left(g_{6}^{2}\right)^{2}+\left(g_{6}^{1 \perp}\right)^{2}\right)\right) \\
& +\frac{1}{\left(2 \pi v_{A}\right)^{2}} g_{3}^{1 \perp}\left(2 g_{1}^{2}\left(g_{1}^{1 \|}-g_{1}^{2}\right)+\left(\left(g_{1}^{1 \|}-g_{1}^{2}\right)^{2}+\left(g_{1}^{2}\right)^{2}+\left(g_{1}^{1 \perp}\right)^{2}\right)+2 g_{3}^{2}\left(g_{3}^{1 \|}-g_{3}^{2}\right)\right. \\
& \left.+\left(\left(g_{3}^{1 \|}-g_{3}^{2}\right)^{2}+\left(g_{3}^{2}\right)^{2}+\left(g_{3}^{1 \perp}\right)^{2}\right)+\left(\left(g_{2}^{1 \|}-g_{2}^{2}\right)^{2}+\left(g_{2}^{1 \perp}\right)^{2}+\left(g_{2}^{2}\right)^{2}\right)\right)+\frac{1}{\left(2 \pi v_{A}\right)^{2}}\left(2 g_{1}^{1 \perp} g_{2}^{2}\left(g_{2}^{1 \|}-g_{2}^{2}\right)\right) \\
\frac{d g_{3}^{2}}{d \ln \left(\omega / E_{0}\right)}= & \frac{1}{2 \pi v_{A}}\left(\left(g_{3}^{1 \perp}\right)^{2}+\left(g_{2}^{2}\right)^{2}+\left(g_{2}^{1 \perp}\right)^{2}\right)+\frac{1}{2 \pi v_{B}}\left(\left(g_{6}^{2}\right)^{2}+\left(g_{6}^{1 \perp}\right)^{2}\right)+\frac{1}{\pi^{2}\left(v_{A}+v_{B}\right)^{2}} g_{3}^{1 \|}\left(g_{5}^{1 \perp}\right)^{2}
\end{aligned}
$$$$
+\frac{1}{\left(2 \pi v_{A}\right)^{2}}\left(g_{3}^{1 \|}\left(\left(g_{1}^{1 \perp}\right)^{2}+\left(g_{3}^{1 \perp}\right)^{2}\right)+g_{1}^{1 \|}\left(g_{2}^{1 \perp}\right)^{2}+\left(g_{3}^{2}-g_{1}^{2}\right)\left(\left(g_{2}^{1 \|}-g_{2}^{2}\right)^{2}+\left(g_{2}^{1 \perp}\right)^{2}+\left(g_{2}^{2}\right)^{2}\right)\right)
$$$$
+\frac{1}{2 \pi^{2} v_{B}\left(v_{A}+v_{B}\right)}\left(g_{5}^{1 \|}\left(g_{6}^{1 \perp}\right)^{2}+\left(g_{3}^{2}-g_{5}^{2}\right)\left(\left(g_{6}^{1 \|}-g_{6}^{2}\right)^{2}+\left(g_{6}^{2}\right)^{2}+\left(g_{6}^{1 \perp}\right)^{2}\right)\right)
$$$$
\begin{aligned}
\frac{d g_{3}^{1 \|}}{d \ln \left(\omega / E_{0}\right)}= & \frac{1}{2 \pi v_{A}}\left(-\left(g_{2}^{1 \|}-g_{2}^{2}\right)^{2}+2\left(g_{3}^{1 \perp}\right)^{2}+\left(g_{2}^{2}\right)^{2}+\left(g_{2}^{1 \perp}\right)^{2}\right)+\frac{1}{2 \pi v_{B}}\left(-\left(g_{6}^{1 \|}-g_{6}^{2}\right)^{2}+\left(g_{6}^{2}\right)^{2}+\left(g_{6}^{1 \perp}\right)^{2}\right) \\
& +\frac{1}{\left(2 \pi v_{A}\right)^{2}}\left(2 g_{3}^{1 \|}\left(\left(g_{1}^{1 \perp}\right)^{2}+\left(g_{3}^{1 \perp}\right)^{2}\right)+\left(g_{3}^{1 \|}-g_{1}^{1 \|}\right)\left(\left(g_{2}^{1 \|}-g_{2}^{2}\right)^{2}+\left(g_{2}^{2}\right)^{2}\right)+\left(g_{3}^{1 \|}+g_{1}^{1 \|}\right)\left(g_{2}^{1 \perp}\right)^{2}\right) \\
& +\frac{1}{\pi^{2}\left(v_{A}+v_{B}\right)^{2}} 2 g_{3}^{1 \|}\left(g_{5}^{1 \perp}\right)^{2}+\frac{1}{2 \pi^{2} v_{B}\left(v_{A}+v_{B}\right)}\left(\left(g_{3}^{1 \|}-g_{5}^{1 \|}\right)\left(\left(g_{6}^{1 \|}-g_{6}^{2}\right)^{2}+\left(g_{6}^{2}\right)^{2}\right)+\left(g_{3}^{1 \|}+g_{5}^{1 \|}\right)\left(g_{6}^{1 \perp}\right)^{2}\right)
\end{aligned}
$$$$
\frac{d g_{4}^{1 \perp}}{d \ln \left(\omega / E_{0}\right)}=\frac{1}{2 \pi v_{A}} 4 g_{6}^{2} g_{6}^{1 \perp}+\frac{1}{2 \pi v_{B}} 2 g_{4}^{1 \perp} g_{4}^{1 \|}+\frac{1}{2 \pi^{2} v_{A}\left(v_{A}+v_{B}\right)} 2\left(2 g_{5}^{1 \perp} g_{6}^{2}\left(g_{6}^{1 \|}-g_{6}^{2}\right)+g_{4}^{1 \perp}\left(\left(g_{6}^{1 \|}-g_{6}^{2}\right)^{2}+\left(g_{6}^{2}\right)^{2}+\left(g_{6}^{1 \perp}\right)^{2}\right)\right)
$$$$
+\frac{1}{\pi^{2}\left(v_{A}+v_{B}\right)^{2}} 2 g_{4}^{1 \perp}\left(\left(g_{5}^{1 \|}\right)^{2}+\left(g_{5}^{1 \perp}\right)^{2}\right)+\frac{1}{\left(2 \pi v_{B}\right)^{2}} g_{4}^{1 \perp}\left(\left(g_{4}^{1 \|}\right)^{2}+\left(g_{4}^{1 \perp}\right)^{2}\right)
$$$$
\frac{d g_{4}^{2}}{d \ln \left(\omega / E_{0}\right)}=\frac{1}{2 \pi v_{A}}\left(2\left(g_{6}^{1 \perp}\right)^{2}+2\left(g_{6}^{2}\right)^{2}\right)+\frac{1}{2 \pi v_{B}}\left(g_{4}^{1 \perp}\right)^{2}
$$$$
+\frac{1}{2 \pi^{2} v_{A}\left(v_{A}+v_{B}\right)} 2\left(g_{5}^{1 \|}\left(g_{6}^{1 \perp}\right)^{2}+\left(g_{4}^{2}-g_{5}^{2}\right)\left(\left(g_{6}^{1 \|}-g_{6}^{2}\right)^{2}+\left(g_{6}^{2}\right)^{2}+\left(g_{6}^{1 \perp}\right)^{2}\right)\right)
$$$$
+\frac{1}{\pi^{2}\left(v_{A}+v_{B}\right)^{2}} 2 g_{4}^{1 \|}\left(g_{5}^{1 \perp}\right)^{2}+\frac{1}{\left(2 \pi v_{B}\right)^{2}}\left(g_{4}^{1 \perp}\right)^{2} g_{4}^{1 \|}
$$$$
\frac{d g_{4}^{1 \|}}{d \ln \left(\omega / E_{0}\right)}=\frac{1}{2 \pi v_{A}}\left(-2\left(g_{6}^{1 \|}-g_{6}^{2}\right)^{2}+2\left(g_{6}^{2}\right)^{2}+2\left(g_{6}^{1 \perp}\right)^{2}\right)+\frac{1}{2 \pi v_{B}} 2\left(g_{4}^{1 \perp}\right)^{2}
$$$$
+\frac{1}{2 \pi^{2} v_{A}\left(v_{A}+v_{B}\right)} 2\left(\left(g_{4}^{1 \|}-g_{5}^{1 \|}\right)\left(\left(g_{6}^{1 \|}-g_{6}^{2}\right)^{2}+\left(g_{6}^{2}\right)^{2}\right)+\left(g_{4}^{1 \|}+g_{5}^{1 \|}\right)\left(g_{6}^{1 \perp}\right)^{2}\right)
$$

$$
\begin{aligned}
& +\frac{1}{\pi^{2}\left(v_{A}+v_{B}\right)^{2}} 4 g_{4}^{1 \|}\left(g_{5}^{1 \perp}\right)^{2}+\frac{1}{\left(2 \pi v_{B}\right)^{2}} 2\left(g_{4}^{1 \perp}\right)^{2} g_{4}^{1 \|} \\
d \ln \left(\omega / E_{0}\right) & =\frac{1}{\pi\left(v_{A}+v_{B}\right)} 2\left(\left(g_{6}^{1 \|}-g_{6}^{2}\right) g_{6}^{1 \perp}+g_{5}^{1 \perp} g_{5}^{1 \|}\right) \\
& +\frac{1}{2 \pi^{2} v_{A}\left(v_{A}+v_{B}\right)}\left(g_{6}^{2}\left(g_{1}^{1 \perp}+g_{3}^{1 \perp}\right)\left(g_{6}^{1 \|}-g_{6}^{2}\right)+g_{5}^{1 \perp}\left(\left(g_{6}^{1 \|}-g_{6}^{2}\right)^{2}+\left(g_{6}^{2}\right)^{2}+\left(g_{6}^{1 \perp}\right)^{2}\right)\right) \\
& +\frac{1}{2 \pi^{2} v_{B}\left(v_{A}+v_{B}\right)}\left(\frac{1}{2}\right)\left(2 g_{4}^{1 \perp} g_{6}^{2}\left(g_{6}^{1 \|}-g_{6}^{2}\right)+g_{5}^{1 \perp}\left(\left(g_{6}^{1 \|}-g_{6}^{2}\right)^{2}+\left(g_{6}^{2}\right)^{2}+\left(g_{6}^{1 \perp}\right)^{2}\right)\right) \\
& +\frac{1}{\left(2 \pi v_{A}\right)^{2}}\left(\frac{1}{2}\right) g_{5}^{1 \perp}\left(\left(g_{2}^{1 \|}\right)^{2}+\left(g_{2}^{1 \perp}\right)^{2}+\left(g_{3}^{1 \|}\right)^{2}+\left(g_{3}^{1 \perp}\right)^{2}+\left(g_{1}^{1 \|}\right)^{2}+\left(g_{1}^{1 \perp}\right)^{2}\right) \\
& +\frac{1}{\left(2 \pi v_{B}\right)^{2}}\left(\frac{1}{2}\right) g_{5}^{1 \perp}\left(\left(g_{4}^{1 \|}\right)^{2}+\left(g_{4}^{1 \perp}\right)^{2}\right)+\frac{1}{\pi^{2}\left(v_{A}+v_{B}\right)^{2}}\left(\frac{3}{2}\right) g_{5}^{1 \perp}\left(\left(g_{5}^{1 \|}\right)^{2}+\left(g_{5}^{1 \perp}\right)^{2}\right)
\end{aligned}
$$




$$
\begin{aligned}
\frac{d g_{5}^{2}}{d \ln \left(\omega / E_{0}\right)}= & \frac{1}{\pi\left(v_{A}+v_{B}\right)}\left(\left(g_{5}^{1 \perp}\right)^{2}-\left(g_{6}^{2}\right)^{2}\right)+\frac{1}{\pi^{2}\left(v_{A}+v_{B}\right)^{2}}\left(\frac{3}{2}\right)\left(g_{5}^{1 \perp}\right)^{2} g_{5}^{1 \|} \\
& +\frac{1}{2 \pi^{2} v_{A}\left(v_{A}+v_{B}\right)}\left(\frac{1}{2}\right)\left(\left(g_{3}^{1 \|}+g_{1}^{1 \|}\right)\left(g_{6}^{1 \perp}\right)^{2}+\left(2 g_{5}^{2}-g_{1}^{2}-g_{3}^{2}\right)\left(\left(g_{6}^{1 \|}-g_{6}^{2}\right)^{2}+\left(g_{6}^{2}\right)^{2}+\left(g_{6}^{1 \perp}\right)^{2}\right)\right) \\
& +\frac{1}{2 \pi^{2} v_{B}\left(v_{A}+v_{B}\right)}\left(\frac{1}{2}\right)\left(g_{4}^{1 \|}\left(g_{6}^{1 \perp}\right)^{2}+\left(g_{5}^{2}-g_{4}^{2}\right)\left(\left(g_{6}^{1 \|}-g_{6}^{2}\right)^{2}+\left(g_{6}^{2}\right)^{2}+\left(g_{6}^{1 \perp}\right)^{2}\right)\right) \\
& +\frac{1}{\left(2 \pi v_{A}\right)^{2}}\left(\frac{1}{2}\right) g_{5}^{1 \|}\left(\left(g_{2}^{1 \perp}\right)^{2}+\left(g_{3}^{1 \perp}\right)^{2}+\left(g_{1}^{1 \perp}\right)^{2}\right)+\frac{1}{\left(2 \pi v_{B}\right)^{2}}\left(\frac{1}{2}\right) g_{5}^{1 \|}\left(g_{4}^{1 \perp}\right)^{2}
\end{aligned}
$$

$$
\begin{aligned}
\frac{d g_{5}^{1 \|}}{d \ln \left(\omega / E_{0}\right)}= & \frac{1}{\pi\left(v_{A}+v_{B}\right)}\left(\left(g_{6}^{1 \|}-g_{6}^{2}\right)^{2}+2\left(g_{5}^{1 \perp}\right)^{2}+\left(g_{6}^{1 \perp}\right)^{2}-\left(g_{6}^{2}\right)^{2}\right)+\frac{1}{\pi^{2}\left(v_{A}+v_{B}\right)^{2}} 3\left(g_{5}^{1 \perp}\right)^{2} g_{5}^{1 \|} \\
& +\frac{1}{2 \pi^{2} v_{A}\left(v_{A}+v_{B}\right)}\left(\frac{1}{2}\right)\left(\left(g_{3}^{1 \|}+g_{1}^{1 \|}\right)\left(-\left(g_{6}^{1 \|}-g_{6}^{2}\right)^{2}-\left(g_{6}^{2}\right)^{2}+\left(g_{6}^{1 \perp}\right)^{2}\right)+2 g_{5}^{1 \|}\left(\left(g_{6}^{1 \|}-g_{6}^{2}\right)^{2}+\left(g_{6}^{2}\right)^{2}+\left(g_{6}^{1 \perp}\right)^{2}\right)\right) \\
& +\frac{1}{2 \pi^{2} v_{B}\left(v_{A}+v_{B}\right)}\left(\frac{1}{2}\right)\left(g_{4}^{1 \|}\left(-\left(g_{6}^{1 \|}-g_{6}^{2}\right)^{2}-\left(g_{6}^{2}\right)^{2}+\left(g_{6}^{1 \perp}\right)^{2}\right)+g_{5}^{1 \|}\left(\left(g_{6}^{1 \|}-g_{6}^{2}\right)^{2}+\left(g_{6}^{2}\right)^{2}+\left(g_{6}^{1 \perp}\right)^{2}\right)\right) \\
& +\frac{1}{\left(2 \pi v_{A}\right)^{2}} g_{5}^{1 \|}\left(\left(g_{2}^{1 \perp}\right)^{2}+\left(g_{3}^{1 \perp}\right)^{2}+\left(g_{1}^{1 \perp}\right)^{2}\right)+\frac{1}{\left(2 \pi v_{B}\right)^{2}} g_{5}^{1 \|}\left(g_{4}^{1 \perp}\right)^{2}
\end{aligned}
$$$$
\frac{d g_{6}^{1 \perp}}{d \ln \left(\omega / E_{0}\right)}=\frac{1}{2 \pi v_{A}}\left(g_{3}^{1 \perp} g_{6}^{2}+g_{2}^{2} g_{6}^{1 \perp}+g_{2}^{1 \perp} g_{6}^{2}+g_{3}^{2} g_{6}^{1 \perp}\right)
$$$$
+\frac{1}{2 \pi v_{B}}\left(g_{4}^{1 \perp} g_{6}^{2}+g_{6}^{1 \perp} g_{4}^{2}\right)+\frac{1}{\pi\left(v_{A}+v_{B}\right)}\left(-2 g_{5}^{2} g_{6}^{1 \perp}-2 g_{5}^{1 \perp} g_{6}^{2}+2 g_{6}^{1 \|} g_{5}^{1 \perp}+2 g_{5}^{1 \|} g_{6}^{1 \perp}\right)
$$$$
+\frac{1}{2 \pi^{2} v_{A}\left(v_{A}+v_{B}\right)} g_{6}^{1 \perp}\left(g_{1}^{1 \|} g_{5}^{2}+g_{1}^{2} g_{5}^{1 \|}-2 g_{1}^{2} g_{5}^{2}+\left(g_{6}^{1 \|}-g_{6}^{2}\right)^{2}+\left(g_{6}^{2}\right)^{2}+\left(g_{6}^{1 \perp}\right)^{2}+g_{3}^{1 \|} g_{5}^{2}+g_{3}^{2} g_{5}^{1 \|}-2 g_{3}^{2} g_{5}^{2}\right)
$$$$
+\frac{1}{\pi^{2}\left(v_{A}+v_{B}\right)^{2}}\left(\frac{3}{2}\right) g_{6}^{1 \perp}\left(\left(g_{5}^{1 \|}-g_{5}^{2}\right)^{2}+\left(g_{5}^{2}\right)^{2}+\left(g_{5}^{1 \perp}\right)^{2}\right)+\frac{1}{\left(2 \pi v_{B}\right)^{2}}\left(\frac{1}{2}\right) g_{6}^{1 \perp}\left(\left(g_{4}^{1 \|}-g_{4}^{2}\right)^{2}+\left(g_{4}^{2}\right)^{2}+\left(g_{4}^{1 \perp}\right)^{2}\right)
$$$$
+\frac{1}{\left(2 \pi v_{A}\right)^{2}}\left(\frac{1}{2}\right) g_{6}^{1 \perp}\left(\left(g_{3}^{1 \|}-g_{3}^{2}\right)^{2}+\left(g_{3}^{2}\right)^{2}+\left(g_{3}^{1 \perp}\right)^{2}+\left(g_{1}^{1 \|}-g_{1}^{2}\right)^{2}+\left(g_{1}^{2}\right)^{2}+\left(g_{1}^{1 \perp}\right)^{2}+\left(g_{2}^{1 \|}-g_{2}^{2}\right)^{2}+\left(g_{2}^{2}\right)^{2}\right.
$$$$
\left.+\left(g_{2}^{1 \perp}\right)^{2}\right)+\frac{1}{2 \pi^{2} v_{B}\left(v_{A}+v_{B}\right)} g_{6}^{1 \perp}\left(g_{5}^{1 \|} g_{4}^{2}+g_{5}^{2} g_{4}^{1 \|}-2 g_{5}^{2} g_{4}^{2}+\left(\frac{1}{2}\right)\left(\left(g_{6}^{1 \|}-g_{6}^{2}\right)^{2}+\left(g_{6}^{2}\right)^{2}+\left(g_{6}^{1 \perp}\right)^{2}\right)\right)
$$

$\frac{d g_{6}^{2}}{d \ln \left(\omega / E_{0}\right)}=\frac{1}{2 \pi v_{A}}\left(\left(g_{3}^{1 \perp}+g_{2}^{1 \perp}\right) g_{6}^{1 \perp}+\left(g_{2}^{2}+g_{3}^{2}\right) g_{6}^{2}\right)+\frac{1}{2 \pi v_{B}}\left(g_{6}^{2} g_{4}^{2}+g_{6}^{1 \perp} g_{4}^{1 \perp}\right)-\frac{1}{\pi\left(v_{A}+v_{B}\right)} 2 g_{6}^{2} g_{5}^{2}+\frac{1}{2 \pi^{2} v_{A}\left(v_{A}+v_{B}\right)} g_{6}^{2}$

$\times\left(-\left(g_{1}^{1 \|}-g_{1}^{2}+g_{3}^{1 \|}-g_{3}^{2}\right)\left(g_{5}^{1 \|}-g_{5}^{2}\right)+\left(g_{6}^{1 \|}-g_{6}^{2}\right)^{2}+\left(g_{6}^{2}\right)^{2}+\left(g_{6}^{1 \perp}\right)^{2}-g_{1}^{1 \perp} g_{5}^{1 \perp}-g_{1}^{2} g_{5}^{2}-g_{3}^{1 \perp} g_{5}^{1 \perp}-g_{3}^{2} g_{5}^{2}\right)$

$+\frac{1}{2 \pi^{2} v_{A}\left(v_{A}+v_{B}\right)} g_{6}^{1 \|} g_{5}^{1 \perp}\left(g_{1}^{1 \perp}+g_{3}^{1 \perp}\right)+\frac{1}{\pi^{2}\left(v_{A}+v_{B}\right)^{2}}\left(\frac{3}{2}\right) g_{6}^{2}\left(\left(g_{5}^{1 \|}-g_{5}^{2}\right)^{2}+\left(g_{5}^{2}\right)^{2}+\left(g_{5}^{1 \perp}\right)^{2}\right)$

$+\frac{1}{\left(2 \pi v_{A}\right)^{2}}\left(\frac{1}{2}\right) g_{6}^{2}\left(\left(g_{3}^{1 \|}-g_{3}^{2}\right)^{2}+\left(g_{3}^{2}\right)^{2}+\left(g_{3}^{1 \perp}\right)^{2}+\left(g_{1}^{1 \|}-g_{1}^{2}\right)^{2}+\left(g_{1}^{2}\right)^{2}+\left(g_{1}^{1 \perp}\right)^{2}+\left(g_{2}^{1 \|}-g_{2}^{2}\right)^{2}+\left(g_{2}^{2}\right)^{2}\right.$

$\left.+\left(g_{2}^{1 \perp}\right)^{2}\right)+\frac{1}{2 \pi^{2} v_{B}\left(v_{A}+v_{B}\right)}\left(g_{6}^{1 \|} g_{5}^{1 \perp} g_{4}^{1 \perp}+g_{6}^{2}\left(-\left(g_{5}^{1 \|}-g_{5}^{2}\right)\left(g_{4}^{1 \|}-g_{4}^{2}\right)-g_{5}^{1 \perp} g_{4}^{1 \perp}-g_{5}^{2} g_{4}^{2}\right.\right.$

$\left.\left.+\left(\frac{1}{2}\right)\left(\left(g_{6}^{1 \|}-g_{6}^{2}\right)^{2}+\left(g_{6}^{2}\right)^{2}+\left(g_{6}^{1 \perp}\right)^{2}\right)\right)\right)+\frac{1}{\left(2 \pi v_{B}\right)^{2}}\left(\frac{1}{2}\right) g_{6}^{2}\left(\left(g_{4}^{1 \|}-g_{4}^{2}\right)^{2}+\left(g_{4}^{2}\right)^{2}+\left(g_{4}^{1 \perp}\right)^{2}\right)$

$$
\begin{aligned}
\frac{d g_{6}^{1 \|}}{d \ln \left(\omega / E_{0}\right)}= & \frac{1}{\pi\left(v_{A}+v_{B}\right)} 2\left(\left(g_{6}^{1 \|}-g_{6}^{2}\right) g_{5}^{1 \|}-g_{5}^{2} g_{6}^{1 \|}+g_{6}^{1 \perp} g_{5}^{1 \perp}\right) \\
& +\frac{1}{2 \pi v_{B}}\left(-g_{6}^{1 \|} g_{4}^{1 \|}+g_{6}^{1 \|} g_{4}^{2}+g_{6}^{2} g_{4}^{1 \|}+g_{6}^{1 \perp} g_{4}^{1 \perp}\right)+\frac{1}{2 \pi v_{A}}\left(-\left(g_{2}^{1 \|}+g_{3}^{1 \|}\right)\left(g_{6}^{1 \|}-g_{6}^{2}\right)\right. \\
& \left.+\left(g_{3}^{2}+g_{2}^{2}\right) g_{6}^{1 \|}+\left(g_{3}^{1 \perp}+g_{2}^{1 \perp}\right) g_{6}^{1 \perp}\right)+\frac{1}{2 \pi^{2} v_{A}\left(v_{A}+v_{B}\right)} g_{6}^{1 \|}\left(g_{1}^{1 \|} g_{5}^{2}+g_{1}^{2} g_{5}^{1 \|}-2 g_{1}^{2} g_{5}^{2}\right. \\
& \left.-g_{1}^{1 \|} g_{5}^{1 \|}+g_{1}^{1 \perp} g_{5}^{1 \perp}+g_{3}^{2} g_{5}^{1 \|}+g_{3}^{1 \|} g_{5}^{2}-2 g_{3}^{2} g_{5}^{2}-g_{3}^{1 \|} g_{5}^{1 \|}+g_{3}^{1 \perp} g_{5}^{1 \perp}+\left(g_{6}^{1 \|}-g_{6}^{2}\right)^{2}+\left(g_{6}^{2}\right)^{2}+\left(g_{6}^{1 \perp}\right)^{2}\right)
\end{aligned}
$$




$$
\begin{aligned}
& +\frac{1}{\pi^{2}\left(v_{A}+v_{B}\right)^{2}}\left(\frac{3}{2}\right) g_{6}^{1 \|}\left(\left(g_{5}^{1 \|}-g_{5}^{2}\right)^{2}+\left(g_{5}^{2}\right)^{2}+\left(g_{5}^{1 \perp}\right)^{2}\right)+\frac{1}{\left(2 \pi v_{B}\right)^{2}}\left(\frac{1}{2}\right) g_{6}^{1 \|}\left(\left(g_{4}^{1 \|}-g_{4}^{2}\right)^{2}+\left(g_{4}^{2}\right)^{2}+\left(g_{4}^{1 \perp}\right)^{2}\right) \\
& +\frac{1}{\left(2 \pi v_{A}\right)^{2}}\left(\frac{1}{2}\right) g_{6}^{1 \|}\left(\left(g_{3}^{1 \|}-g_{3}^{2}\right)^{2}+\left(g_{3}^{2}\right)^{2}+\left(g_{3}^{1 \perp}\right)^{2}+\left(g_{1}^{1 \|}-g_{1}^{2}\right)^{2}+\left(g_{1}^{2}\right)^{2}+\left(g_{1}^{1 \perp}\right)^{2}+\left(g_{2}^{1 \|}-g_{2}^{2}\right)^{2}+\left(g_{2}^{2}\right)^{2}+\left(g_{2}^{1 \perp}\right)^{2}\right) \\
& +\frac{1}{2 \pi^{2} v_{B}\left(v_{A}+v_{B}\right)} g_{6}^{1 \|}\left(g_{5}^{1 \|} g_{4}^{2}+g_{5}^{2} g_{4}^{1 \|}-2 g_{5}^{2} g_{4}^{2}-g_{5}^{1 \|} g_{4}^{1 \|}+g_{5}^{1 \perp} g_{4}^{1 \perp}+\left(\frac{1}{2}\right)\left(\left(g_{6}^{1 \|}-g_{6}^{2}\right)^{2}+\left(g_{6}^{2}\right)^{2}+\left(g_{6}^{1 \perp}\right)^{2}\right)\right)
\end{aligned}
$$

\section{The scaling of response functions}

Our aim is to obtain response functions that describe the instabilities, because the response function ultimately decides the state of the system. There are four types of response functions: CDW $\overline{N_{n}}$, spin density wave (SDW) $\overline{\chi_{n}}$, singlet superconductivity (SS) $\overline{\Delta_{s, n}}$, and triplet superconductivity (TS) $\overline{\Delta_{t, n}}$. The competition among these response functions determines the ground state in the low energy limit. Following the formalism presented in the literature, ${ }^{52-58}$ we write down the scaling equation of the response functions in channel $n$ up to the first order:

$$
\begin{gathered}
\frac{d \ln \overline{N_{n}}\left(\omega / E_{0}\right)}{d \ln \left(\omega / E_{0}\right)}=\frac{2}{\pi\left(v_{F m}+v_{F m^{\prime}}\right)}\left(g_{n}^{1 \perp}+g_{n}^{1 \|}-g_{n}^{2}\right) \\
\frac{d \ln \overline{\chi_{n}}\left(\omega / E_{0}\right)}{d \ln \left(\omega / E_{0}\right)}=\frac{2}{\pi\left(v_{F m}+v_{F m^{\prime}}\right)}\left(-g_{n}^{2}\right) \\
\frac{d \ln \overline{\Delta_{s, n}}\left(\omega / E_{0}\right)}{d \ln \left(\omega / E_{0}\right)}=\frac{2}{\pi\left(v_{F m}+v_{F m^{\prime}}\right)}\left(g_{n}^{1 \perp}+g_{n}^{2}\right) \\
\frac{d \ln \overline{\Delta_{t, n}}\left(\omega / E_{0}\right)}{d \ln \left(\omega / E_{0}\right)}=\frac{2}{\pi\left(v_{F m}+v_{F m^{\prime}}\right)}\left(-g_{n}^{1 \|}+g_{n}^{2}\right),
\end{gathered}
$$

where Eqs. (7a), (7b), (7c), and (7d) are related to the response functions of CDW, SDW, SS and TS, respectively. They yield the power-law exponents of the response functions, which are only the right sides of the relevant equations. The response function with the largest power-law exponent dominates as the energy scales to zero. It should be noted that Eqs. (2) and (7) are evaluated simultaneously so that the right-hand sides of Eq. (7) are continuously replaced by the latest renormalized coupling constant obtained from Eq. (2). When the coupling constants are renormalized to their respective fixed-point values, then the response functions would approach a power law as a function of $\omega / E_{0}$, in which the slope of $\ln ($ response function) versus $\ln \left(\omega / E_{0}\right)$ is precisely given by the fixed-point values of the coupling constants.

\section{Fixed points}

There are two approaches to obtaining the fixed points. One is to explicitly solve the scaling equations so as to obtain all the fixed points of the coupling. That can be difficult and, in addition, not all the fixed points are relevant. The other approach is to use initial values of the coupling constant and then to integrate the scaling equations. This is the approach we shall follow. ${ }^{59}$

The 1D Coulomb repulsion in momentum space can be written as ${ }^{59,62}$

$$
V_{C} \approx \frac{2 e^{2}}{\kappa} \ln \left(\frac{k_{c}+k}{k}\right),
$$

where $e$ is the electron charge, $\kappa$ denotes the dielectric constant of the medium, with $k$ being the momentum transfer in the scattering process and $k_{c}$ the inverse of CNT radius $R$. The strength of interaction can be expressed in units of dimensionless ratio $e^{2} /\left(2 \pi v_{F} \kappa\right)$. Since $v_{F A}$ is $\sim 2.8 \times 10^{5} \mathrm{~ms}^{-1}$, and $e^{2} / c=1 / 137$, we get $e^{2} /\left(2 \pi v_{F} \kappa\right) \approx 8 / 2 \pi \kappa$. In forward scattering, Eq. (8) has to be estimated with a cutoff at small $k$, with $k \sim 10^{-3} k_{c}$. Therefore, the initial values of the forward scatterings coupling constants $\widetilde{g}_{1}^{2}, \widetilde{g}_{2}^{2}, \widetilde{g}_{4}^{2}, \widetilde{g}_{5}^{2}$ (note that $\widetilde{g}_{n}^{j}=g_{n}^{j} /\left(\pi\left(v_{F m}+v_{F m^{\prime}}\right)\right), n$ being the channel index and $j$ the coupling-type index) with zero-momentum transfer are $\frac{V_{C}}{2 \pi v_{F}} \approx \frac{e^{2}}{2 \pi v_{F} K} 2 \ln \left(\frac{k_{c}}{10^{-3} k_{c}}\right) \sim 15.9 / \kappa$. Other Coulomb repulsive interactions can be evaluated in a similar manner.

The phonon mediated electron-electron coupling is in the form

$$
V_{i j i^{\prime} j^{\prime}}=-2 g_{i i^{\prime}} g_{j j^{\prime}} \frac{\omega_{k}}{-\omega^{2}+\omega_{k}^{2}},
$$

where $g_{i i^{\prime}}, g_{j j^{\prime}}$ are the electron-phonon coupling constants, and $\omega_{k}$ is the phonon frequency. The phonon mediated electronelectron interaction, with momentum transfer $2 k_{F A}$, is given by

$$
\sum_{\nu} 2\left(g_{A A}^{(\nu)}\left(2 k_{F A}\right)\right)^{2} / 2 \pi v_{F A} \omega_{2 k_{F A}}^{(\nu)} \approx 0.095
$$

for the $A A$ channel, where the summation runs over different phonon branches denoted by $v$. For the $B B$ channel the same is given by

$$
\sum_{\nu} 2\left(g_{B B}^{(\nu)}\left(2 k_{F B}\right)\right)^{2} / 2 \pi v_{F A} \omega_{2 k_{F B}}^{(\nu)} \approx 0.175 .
$$

For the $\widetilde{g}_{3}^{2}, \widetilde{g}_{3}^{1 \perp}, \widetilde{g}_{5}^{1 \perp}, \widetilde{g}_{6}^{1 \perp}$, the value is 0.0796 .

It follows that for a single $(5,0) \mathrm{CNT}$, the initial values of the coupling constants are as follows:

$$
\begin{gathered}
\widetilde{g}_{1}^{1 \perp}, \widetilde{g}_{2}^{1 \perp}=-0.095+0.573 / \kappa, \\
\widetilde{g}_{1}^{2}, \widetilde{g}_{2}^{2}, \widetilde{g}_{4}^{2}, \widetilde{g}_{5}^{2}=15.9 / \kappa, \\
\widetilde{g}_{4}^{1 \perp}=-0.175+0.175 / \kappa, \\
\widetilde{g}_{5}^{1 \perp}, \widetilde{g}_{6}^{1 \perp}=-0.0796+0.0796 / \kappa, \\
\widetilde{g}_{6}^{2}=-0.0796+0.0478 / \kappa, \\
\widetilde{g}_{3}^{2}, \widetilde{g}_{3}^{1 \perp}=-0.0796+0.0079 / \kappa .
\end{gathered}
$$

It should be noted that if not indicated, the spin-parallel backward-scattering coupling constant $\widetilde{g}_{n}^{1 \|}$ always equals to antiparallel ones, $\tilde{g}_{n}^{1 \perp}$, in the whole scaling process.

The scaling result is noted to depend on the dielectric constant $\kappa$. If $\kappa=1$, the fixed-point values for a single $(5,0)$ 
CNT case are

$$
\begin{aligned}
\widetilde{g}_{1}^{2} & \rightarrow 16.05, \\
\widetilde{g}_{2}^{2}, \widetilde{g}_{4}^{2} & \rightarrow 15.55, \\
\widetilde{g}_{3}^{2} & \rightarrow-0.5, \\
\widetilde{g}_{5}^{1 \perp} & \rightarrow-2 / 3, \\
\widetilde{g}_{5}^{2} & \rightarrow 15.71, \\
\widetilde{g}_{6}^{1 \perp} & \rightarrow 0.76,
\end{aligned}
$$

and all others are zero. In this case the CDW and SDW response functions dominate. While if $\kappa>1.11$, which is in the range of the AFI medium, the fixed-point values are

$$
\begin{aligned}
& \widetilde{g}_{1}^{1 \perp}, \widetilde{g}_{5}^{1 \perp} \rightarrow 0, \\
& \widetilde{g}_{1}^{2}, \widetilde{g}_{5}^{2} \rightarrow 0.268+15.03 /(\kappa-0.064) \text {, } \\
& \tilde{g}_{2}^{2}, \widetilde{g}_{4}^{2} \rightarrow \widetilde{g}_{1}^{2}-1,
\end{aligned}
$$

while all others are -1 . In this case channels 1 and 5 scale to the LL fixed point if considered by themselves, and in other channels the system scales to strong coupling. Since all six channels are coupled, they necessarily scale to the strong coupling fixed point obtained by the second order RG calculation. It is at this fixed point that the dominant response function depends on the value of $\kappa$. If $\kappa<22$ the CDW order is the most favored, and if $\kappa>22$, the SS order is the most favored.

If a $(5,0) \mathrm{CNT}$ is embedded in a thin array consisting of six other $(5,0)$ CNTs, the situation is different from that of a single $(5,0)$ CNT. As mentioned previously, besides the intratube interactions, there are intertube ones that participate in the scaling equation. In addition, the screening of the Coulomb interaction will influence the initial value, which in turn would influence the resulting fixed-point values. As described in Ref. 59, the screening effect reduces the initial value of forward-scattering couplings. In what follows, we shall approximate the Coulomb interaction between two nearestneighbor CNTs in a thin array by that in an infinite periodic array. The initial values obtained from such an approximation will then be used to evaluate the fixed points of the thin array.

In a periodic CNT array, the partially Fourier-transformed Coulomb potential along the CNT's $c$-axis direction is given by

$$
V_{l, l^{\prime}}(k)=\frac{2 e^{2}}{\kappa} K_{0}\left(k\left|l-l^{\prime}\right|\right),
$$

where $K_{0}(x)$ denotes the modified Bessel function of zeroth order, and $l$ and $l^{\prime}$ denote the positions of the two CNTs along the transverse direction of the bundle. The modified Bessel function diverges logarithmically when $x \rightarrow 0$ and decays exponentially as $x>1$. The potential shown in Eq. (12) serves as the input to the calculation of the screened potential, which can be derived from the RPA approximation with the following Dyson equation:

$$
V_{l, l^{\prime}}^{R P A}(k)=V_{l, l^{\prime}}(k)+\Pi \sum_{l^{\prime \prime} \in \text { bundle }} V_{l, l^{\prime \prime}}(k) V_{l^{\prime \prime}, l^{\prime}}^{R P A}(k),
$$

where $\Pi$ is the polarizability of 1D Fermi gas,

$$
\Pi(k)=\frac{2}{L} \sum_{q} \frac{f\left(\varepsilon_{q+k}\right)-f\left(\varepsilon_{q}\right)}{\varepsilon_{q+k}-\varepsilon_{q}},
$$

with $f$ being the Fermi-Dirac function and $L$ the length of CNT. By performing a 2D Fourier transform of $V_{l, l^{\prime}}(k)$ and $V_{l, l^{\prime}}^{R P A}(k)$ along the transverse direction of the periodic array, we have

$$
V_{l, l^{\prime}}(\vec{k})=\left(\frac{d}{2 \pi}\right)^{2} \int_{B Z} d^{2} \vec{p} \Phi(\vec{k}, \vec{p}) e^{i \vec{p} \cdot\left(l-l^{\prime}\right)},
$$

in which $B Z$ means integration over the first Brillouin zone of the transverse lattice of CNT arrays. Here $d$ denotes the lattice constant between two nearest-neighbor CNTs, which in our case is about $1 \mathrm{~nm}$. Then the Fourier-transformed potentials $\Phi$ and $\Phi^{R P A}$ obey a simplified Dyson function:

$$
\Phi^{R P A}(\vec{k}, \vec{p})=\Phi(\vec{k}, \vec{p})+\Pi(\vec{k}) \Phi(\vec{k}, \vec{p}) \Phi^{R P A}(\vec{k}, \vec{p}) .
$$

Finally one can write down the screened Coulomb potential with momentum transfer $k$ along the tube-axis direction between two electrons located on tubes $l$ and $l^{\prime}$ :

$V_{l, l^{\prime}}^{R P A}(\vec{k})=\left(\frac{d}{2 \pi}\right)^{2} \int_{B Z} d^{2} \vec{p} \frac{\Phi(\vec{k}, \vec{p})}{1-\Pi(\vec{k}) \Phi(\vec{k}, \vec{p})} e^{i \vec{p} \cdot\left(l-l^{\prime}\right)}$.

Following this method, we get the initial values of the intratube (with $l=l^{\prime}$ ) forward-scattering coupling constants in the presence of intertube coupling, which are

$$
\widetilde{g}_{1}^{2}=\widetilde{g}_{2}^{2}=\widetilde{g}_{4}^{2}=\widetilde{g}_{5}^{2} \sim 0.1,
$$

where it is seen that the screening can efficiently reduce the initial value of the Coulomb repulsion.

The new intertube couplings' initial values (denoted with upper bar to distinguish from intratube couplings), with $\mid l-$ $l^{\prime} \mid=d \sim 1 \mathrm{~nm}$, are given by

$$
\begin{aligned}
& \overline{\widetilde{g}_{1}^{2}}=\overline{\widetilde{g}_{2}^{2}}=0.00032 \kappa, \\
& \overline{\widetilde{g}_{1}^{1 \perp}}=\overline{\widetilde{g}_{2}^{1 \perp}}=0.045 / \kappa .
\end{aligned}
$$

The fixed points are also dependent on the dielectric constant. If $\kappa=1$, the fixed points are given by

$$
\begin{gathered}
\widetilde{g}_{1}^{1 \perp}, \widetilde{g}_{2}^{1 \perp}, \widetilde{g}_{4}^{1 \perp} \rightarrow 1, \\
\tilde{g}_{1}^{1 \|}, \widetilde{g}_{2}^{1 \|}, \widetilde{g}_{4}^{1 \|} \rightarrow-1, \\
\widetilde{g}_{4}^{2} \rightarrow-0.42628, \\
\frac{\widetilde{g}_{1}^{1 \perp}, \overline{g_{2}}}{\widetilde{g}^{1 \perp}} \rightarrow 0.40825, \\
\widetilde{g}_{1}^{1 \|}, \overline{\sigma_{2}^{1 \|}} \rightarrow-0.40825,
\end{gathered}
$$

while other coupling constants scale to zero. In this case $\widetilde{g}_{n}^{1 \perp}$ and $\widetilde{g}_{n}^{1 \|}$ are no longer identical to each other. All the response functions remain small, and the largest among them, when scaling to low energy limit, is the CDW in channel 5, shown in Fig. $1(\mathrm{~g})$. When $\kappa>1.38$, then the fixed points are similar to the case of a single CNT case at $\kappa>1.11$, with the fixed-point value

$$
\widetilde{g}_{1}^{2}, \widetilde{g}_{5}^{2} \rightarrow 0.287
$$


when $\kappa=3$, while the largest response function is the SS type in the $\widetilde{g}_{6}^{1 \|, 1 \perp, 2}, \widetilde{g}_{4}^{1 \|, 1 \perp, 2}, \widetilde{g}_{3}^{1 \|, 1 \perp, 2}$, and $\widetilde{g}_{2}^{1 \|, 1 \perp, 2}$ channels. The calculated fixed points for the SS channel, where it is noted that

$$
\frac{2}{\pi\left(v_{F m}+v_{F m^{\prime}}\right)}\left(g_{n}^{1 \perp}+g_{n}^{2}\right)=2\left(\widetilde{g}_{n}^{1 \perp}+\widetilde{g}_{n}^{2}\right),
$$

are respectively, $0.573,-4,-3.427,-3.427,0.573$, and -4 in the six channels. For the CDW it is noted that

$$
\frac{2}{\pi\left(v_{F m}+v_{F m^{\prime}}\right)}\left(g_{n}^{1 \perp}+g_{n}^{1 \|}-g_{n}^{2}\right)=2\left(\widetilde{g}_{n}^{1 \perp}+\widetilde{g}_{n}^{1 \|}-\widetilde{g}_{n}^{2}\right),
$$

and the fixed-point values are $-0.573,-2,-2.573,-2.573$, -0.573 , and -2 , respectively. Therefore, the most divergent response function is now the $\mathrm{SS}$ in the channels $\widetilde{g}_{2}^{1 \|, 1 \perp, 2}$ and $\tilde{g}_{6}^{1 \|, 1 \perp, 2}$. SDW and TS are less divergent and therefore not as important. This result can be attributed to the small initial values of $\widetilde{g}_{1}^{2}$ and $\widetilde{g}_{5}^{2}$, which imply that the screening effect plays an important role in the appearance of superconductivity. Another interesting feature is the crossover between the CDW and SS response functions during the scaling to the low energy limit, as shown in Fig. 5. This crossover means ${ }^{63}$ that at the beginning of the scaling process the CDW response in the $\widetilde{g}_{3}^{1 \|, 1 \perp, 2}, \widetilde{g}_{4}^{1 \|, 1 \perp, 2}$, and $\widetilde{g}_{5}^{1 \|, 1 \perp, 2}$ channels (CDW in channel $\widetilde{g}_{4}^{1 \|, 1 \perp, 2}$ is the largest) dominate, but as scaling proceeds to lower energies the SS response functions eventually wins because it is the most divergent. In Fig. 6 we summarize in the form of a phase diagram, plotted as a function of the dielectric constant; the results are obtained from our calculations. In Fig. 6(a) the phase diagram is for a single $(5,0) \mathrm{CNT}$, whereas in Fig. 6(b) the phase diagram is for a thin array of $(5,0)$ CNTs comprising a central nanotube surrounded by six others, with a separation of $\sim 1 \mathrm{~nm}$. It is seen clearly that for the thin array case the superconducting phase is favored at a much smaller value of the dielectric constant $\kappa$, mainly owing to the screening effect on the Coulomb interaction offered by the neighboring nanotubes. Our results thus support the appearance of superconductivity in 4-Angstrom CNTs embedded in an AFI zeolite matrix, which has a $\kappa=6$.

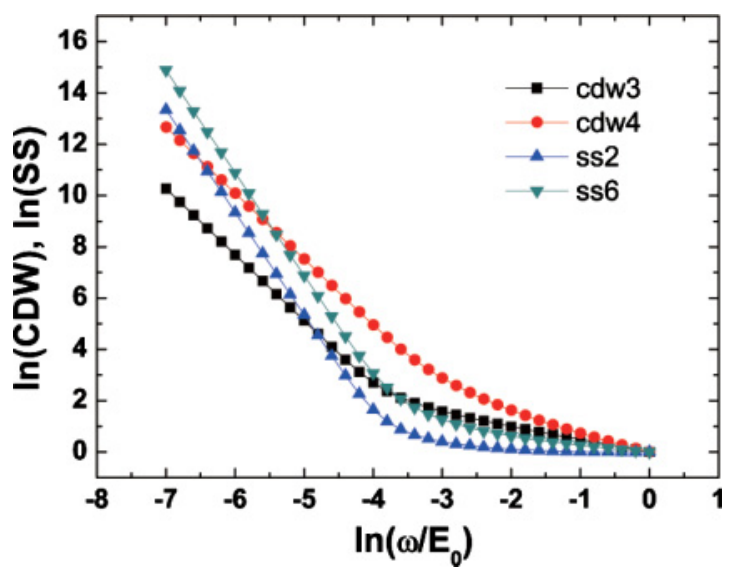

FIG. 5. (Color online) The crossover of the CDW and SS orders. Only the two most divergent response functions of CDW and SS are shown.
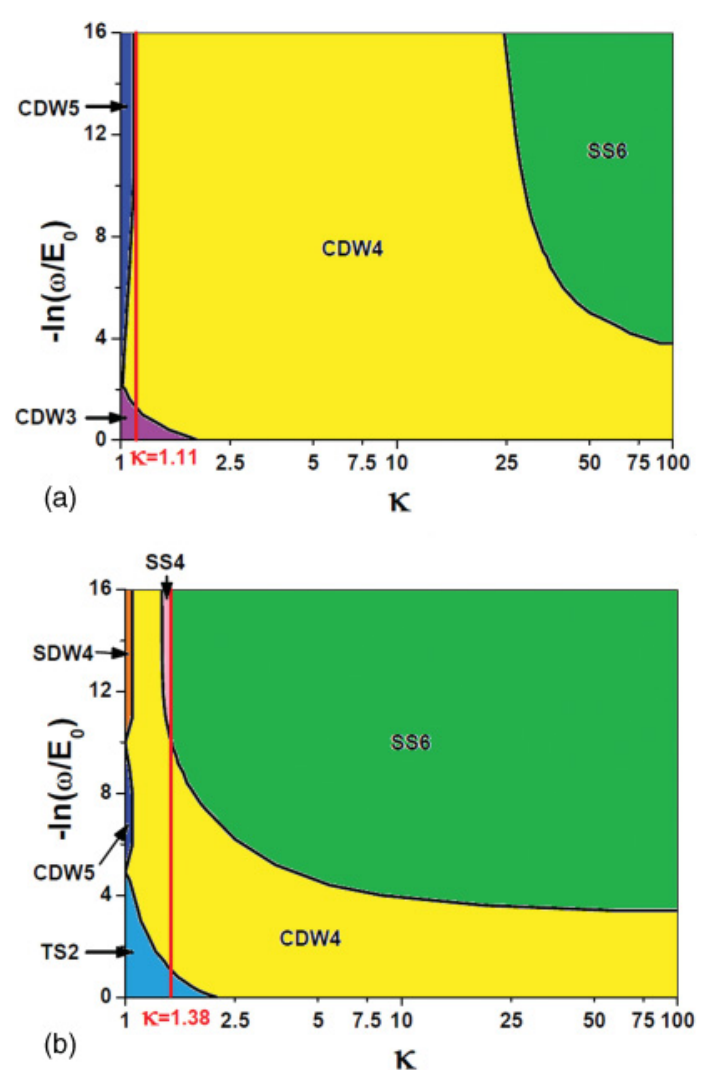

FIG. 6. (Color online) The phase diagram of the most dominant response function of the $(5,0)$ electronic system plotted as a function of the dielectric constant $\kappa$ of the embedding matrix, as analyzed by the second-order renormalization group calculations. The case of a single $(5,0)$ CNT is shown in (a), and in (b) the case of a thin array of $(5,0) \mathrm{CNTs}$, comprising one central nanotube surrounded by six others with a separation of $1 \mathrm{~nm}$, is shown. Here the $Y$-axis denotes the scaling factor $-\ln \left(\omega / E_{0}\right)$. Increasing $Y$ implies decreasing energy/temperature. Different phases are delineated by different colors/shades of gray, with the number following the abbreviation for each phase denoting the divergent channel. For example, "CDW4" in the central part of Fig. 6(a) means that in this region, the largest response function is the CDW order in the fourth channel, etc. The vertical lines at $\kappa=1.11$ and $\kappa=1.38$ mark the division between different fixed points. Within the array configuration, the SS order is favored over a large range of dielectric constant $\kappa$. In particular, for the AFI zeolite with $\kappa=6$, superconducting ground state is favored.

\section{DEVICE MANIFESTATION OF THE CDW STATE}

In order to experimentally observe the state of the CNT thin array, it is generally necessary to make electrode contact to the sample. Hence the device behavior necessarily involves the electrodes. Here we deduce the manifestation of the CDW order in anticipation of our interpretation of the observed experimental results.

We have performed tunneling-resistance calculations by using the Landauer-Buttiker formula. ${ }^{64,65}$ Consider a 1D Fermi gas in contact with two leads at $x_{1}$ and $x_{2}\left(x_{1}<x_{2}\right)$; the self-energy of the contact can be expressed as

$$
\Sigma_{L, R}(x, \omega)=\sum_{n \in \text { lead }}\left|V_{k, n}(x)\right|^{2} \frac{1}{\omega-\varepsilon_{n}},
$$



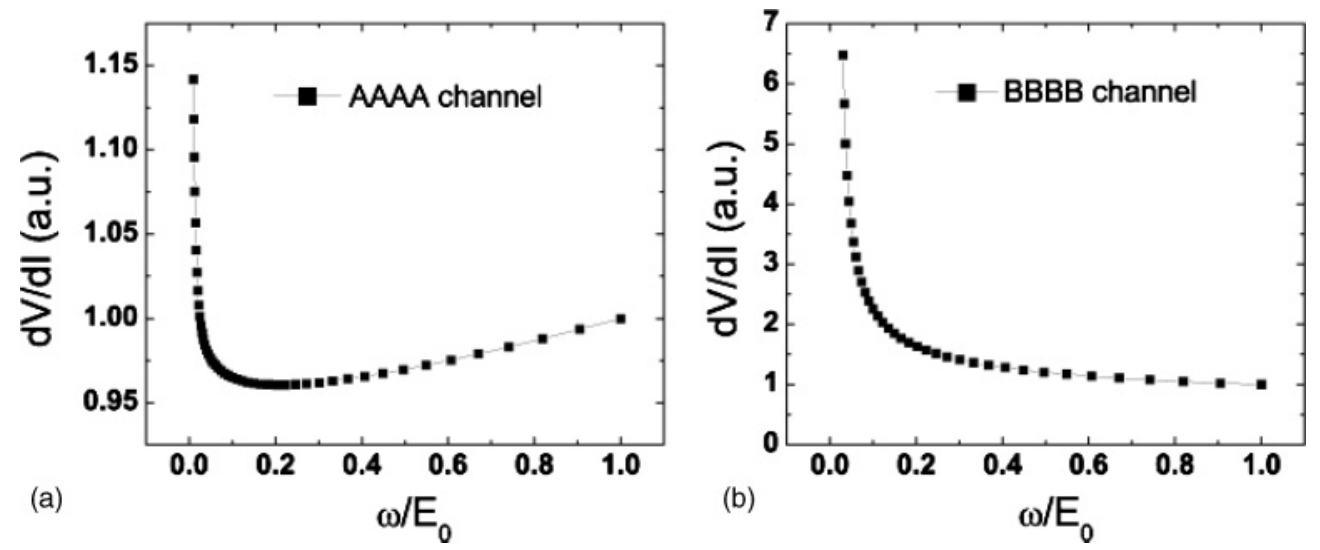

FIG. 7. (a) and (b) are the calculated differential resistance versus the energy-scaling variable in channels 1 and 4 . Both are characterized by a peak in differential resistance at the Fermi level.

where $V$ is the tunneling potential between the 1D channel and the lead, $k$ denotes the states in the 1D Fermi gas, $n$ denotes the states in the lead, $\Sigma$ denotes the self-energy, and $L$ and $R$ denote left and right leads, respectively. This self-energy can be incorporated in the Hamiltonian $H_{0}+H_{\text {int }}$, and a scaling analysis can be performed. ${ }^{65,66}$ First we write down the eigenfunctions of $H_{0}$,

$$
\begin{aligned}
\phi_{k}(x) & =\frac{1}{\sqrt{2 \pi}} e^{i k x}, \\
\phi_{-k}(x) & =\frac{1}{\sqrt{2 \pi}} e^{-i k x},
\end{aligned}
$$

around the two Fermi points. Then owing to the self-energy of the electrode, the new wave functions can be evaluated with the Dyson equation:

$$
\begin{gathered}
\psi_{k}(x)=\frac{1}{\sqrt{2 \pi}} \begin{cases}e^{i k x}+e^{-i k x} \Sigma_{L, k,-k}+e^{-i k x} \Sigma_{R, k,-k} & x<x_{1} \\
e^{i k x}+e^{i k x} \Sigma_{L, k, k}+e^{-i k x} \Sigma_{R, k,-k} & x_{1}<x<x_{2}, \\
e^{i k x}+e^{i k x} \Sigma_{L, k, k}+e^{i k x} \Sigma_{R, k, k} & x>x_{2}\end{cases} \\
\psi_{-k}(x)=\frac{1}{\sqrt{2 \pi}} \begin{cases}e^{-i k x}+e^{-i k x} \Sigma_{L,-k,-k}+e^{-i k x} \Sigma_{R,-k,-k} & x<x_{1} \\
e^{-i k x}+e^{i k x} \Sigma_{L, k,-k}+e^{-i k x} \Sigma_{R,-k,-k} & x_{1}<x<x_{2}, \\
e^{-i k x}+e^{i k x} \Sigma_{L, k,-k}+e^{i k x} \Sigma_{R, k,-k} & x>x_{2}\end{cases}
\end{gathered}
$$

where $x_{1}, x_{2}$ denote the positions of the electrodes. It can be shown that the previous basis functions directly diagonalize the lead self-energy in conjunction with $H_{0}$. Hence it would be convenient to express the electron-electron interaction part of the Hamiltonian, $H_{\mathrm{int}}$, in this new basis. For simplicity we assume the electrode to be a point-contact. We define annihilation operators $a_{k}, b_{k}$ that destroy an electron for the wave functions shown in Eqs. (19) and (20), respectively, while $c_{q}, d_{q}$ destroy an electron for the wave functions shown in Eqs. (21) and (22), respectively. By making a transformation of the four operators as

$$
a_{k \sigma}=\int d q\left[A_{k}^{q \sigma} c_{q \sigma}+B_{k}^{q \sigma} d_{q \sigma}\right], \quad b_{k \sigma}=\int d q\left[C_{k}^{q \sigma} d_{q \sigma}+D_{k}^{q \sigma} c_{q \sigma}\right],
$$

and by taking $x_{1} \rightarrow-\infty$ and $x_{2} \rightarrow \infty$, we find

$$
\begin{array}{ll}
A_{k}^{q \sigma}=T_{L}\left[\frac{i}{k-q+i \delta}-\frac{i}{k-q-i \delta}\right], & B_{k}^{q \sigma}=R_{L}\left[\frac{i}{k-q+i \delta}-\frac{i}{k-q-i \delta}\right] \\
C_{k}^{q \sigma}=T_{R}\left[\frac{i}{k-q+i \delta}-\frac{i}{k-q-i \delta}\right], & D_{k}^{q \sigma}=R_{R}\left[\frac{i}{k-q+i \delta}-\frac{i}{k-q-i \delta}\right],
\end{array}
$$

in which we have defined $T_{L}=1+\Sigma_{L, k, k}, R_{L}=\Sigma_{L, k,-k}, T_{R}=1+\Sigma_{R, k, k}$, and $R_{R}=\Sigma_{R, k,-k}$.

Consider the electron-electron interaction Hamiltonian,

$$
H_{\mathrm{int}}=\frac{1}{2 \pi} \int d k d p d q \sum_{\sigma \sigma^{\prime}}\left[g_{1} a_{k \sigma}^{+} b_{-p \sigma^{\prime}}^{+} a_{p+2 k f+q, \sigma^{\prime}} b_{-k+2 k f+q, \sigma}+g_{2} a_{k \sigma}^{+} b_{-p \sigma^{\prime}}^{+} b_{-p-q, \sigma^{\prime}} a_{k-q, \sigma}\right] .
$$


We find that it can also be expressed under the new basis as

$$
\begin{aligned}
H_{\mathrm{back}}= & \frac{1}{2 \pi} \int d k d p d q \int d q_{1} d q_{2} d q_{3} d q_{4} \sum_{\sigma \sigma^{\prime}}\left[g_{1}\left(A_{k}^{q_{1} \sigma+} c_{q_{1} \sigma}^{+}+B_{k}^{q_{1} \sigma+} d_{q_{1} \sigma}^{+}\right)\left(C_{-p}^{q_{2} \sigma^{\prime}+} d_{q_{2} \sigma^{\prime}}^{+}+D_{-p}^{q_{2} \sigma^{\prime}+} c_{q_{2} \sigma^{\prime}}^{+}\right)\right. \\
& \left.\times\left(A_{p+2 k f+q}^{q_{3}, \sigma^{\prime}} c_{q_{3}, \sigma^{\prime}}+B_{p+2 k f+q}^{q_{3}, \sigma^{\prime}} d_{q_{3}, \sigma^{\prime}}\right)\left(C_{-k+2 k f+q}^{q_{4}, \sigma} d_{q_{4}, \sigma}+D_{-k+2 k f+q}^{q_{4}, \sigma} c_{q_{4}, \sigma}\right)\right], \\
H_{\text {forward }}= & \frac{1}{2 \pi} \int d k d p d q \int d q_{1} d q_{2} d q_{3} d q_{4} \sum_{\sigma \sigma^{\prime}}\left[g_{2}\left(A_{k}^{q_{1} \sigma+} c_{q_{1} \sigma}^{+}+B_{k}^{q_{1} \sigma+} d_{q_{1} \sigma}^{+}\right)\left(D_{-p}^{q_{2} \sigma^{\prime}+} c_{q_{2} \sigma^{\prime}}^{+}+C_{-p}^{q_{2} \sigma^{\prime}+} d_{q_{2} \sigma^{\prime}}^{+}\right)\right. \\
& \left.\times\left(D_{-p-q}^{q_{3} \sigma^{\prime}} c_{q_{3} \sigma^{\prime}}+C_{-p-q}^{q_{3} \sigma^{\prime}} d_{q_{3} \sigma^{\prime}}\right)\left(A_{k-q}^{q_{4} \sigma} c_{q_{4} \sigma}+B_{k-q}^{q_{4} \sigma} d_{q_{4} \sigma}\right)\right] .
\end{aligned}
$$

Consider a reduction of the bandwidth from $E_{0}$ to $E_{0}-\delta E_{0}$. By performing a scaling $52,65,66$ on the Hamiltonian, we get a new Hamiltonian which is defined on a narrower energy band by eliminating degrees of freedom in the energy range $\left(E_{0}-\delta E_{0}, E_{0}\right)$. This is accomplished by replacing the product of two Fermi operators by their averaged value in this range. In the presence of leads there are nondiagonal parts in the interaction Hamiltonian,

$$
\begin{aligned}
H_{\text {int }}= & \text { const } * \int d p d k \frac{2 \delta E_{0}}{E_{0}+\varepsilon(k)+\varepsilon(q)} \sum_{\sigma \sigma^{\prime}}\left(g_{1}-g_{2} \delta_{\sigma \sigma^{\prime}}\right) \\
& \times\left[\left(T_{L}^{\sigma *} R_{R}^{\sigma / *} T_{L}^{\sigma \prime} T_{R}^{\sigma}+T_{L}^{\sigma *} R_{R}^{\sigma *} R_{L}^{\sigma \prime} R_{R}^{\sigma}+T_{L}^{\sigma *} T_{R}^{\sigma *} R_{L}^{\sigma \prime} T_{R}^{\sigma}+R_{L}^{\sigma *} R_{R}^{\sigma *} R_{L}^{\sigma \prime} T_{R}^{\sigma}\right) c_{k \sigma}^{+} d_{q, \sigma}\right. \\
& \left.+\left(R_{L}^{\sigma *} R_{R}^{\sigma / *} T_{L}^{\sigma \prime} R_{R}^{\sigma}+T_{L}^{\sigma *} T_{R}^{\sigma / *} T_{L}^{\sigma \prime} R_{R}^{\sigma}+R_{L}^{\sigma *} T_{R}^{\sigma * *} T_{L}^{\sigma \prime} T_{R}^{\sigma}+R_{L}^{\sigma *} T_{R}^{\sigma *} R_{L}^{\sigma \prime} R_{R}^{\sigma}\right) d_{k \sigma}^{+} c_{q \sigma}\right] .
\end{aligned}
$$

From Eq. (18) we can see that the self-energy $\Sigma_{\mathrm{k}, \mathrm{k}^{\prime}}$ depends on the tunneling potential $V$ that connects states inside the 1D Fermi system with those states in the electrode. We can assume this tunneling to be independent of $k$ so that $V_{k, n}=V_{-k, n}$, where $n$ denotes states inside the electrode. It follows from Eq. (14) that $\Sigma_{k, k}=\Sigma_{k,-k}$. If in addition we assume the leads to be symmetric, i.e., $R_{L}=R_{R}, T_{L}=T_{R}$, then the Hamiltonian can be renormalized back to its original diagonal form by transforming $T_{\sigma} \rightarrow T_{\sigma}+\delta T_{\sigma}$ (and similarly for $R_{\sigma}$ since they are related). The necessary $\delta T_{\sigma}$ that will do the job is given by

$$
\delta T_{\sigma}=\left[\frac{R_{\sigma}^{*} T_{\sigma}\left(T_{\sigma} T_{\sigma}^{*}+R_{\sigma} R_{\sigma}^{*}\right)}{\left[\left(T_{\sigma}+R_{\sigma}\right)\left(T_{\sigma}^{*}+R_{\sigma}^{*}\right)^{2}\right]}\left(\frac{g_{2}-g_{1 \|}}{2 \pi v_{F}}\right)-\frac{R_{\sigma}^{*} T_{\sigma}\left(T_{-\sigma} T_{-\sigma}^{*}+R_{-\sigma} R_{-\sigma}^{*}\right)}{\left[\left(T_{-\sigma}+R_{-\sigma}\right)\left(T_{\sigma}^{*}+R_{\sigma}^{*}\right)\left(T_{-\sigma}^{*}+R_{-\sigma}^{*}\right)\right]}\left(\frac{g_{1 \perp}}{2 \pi v_{F}}\right)\right] \frac{2 \delta E_{0}}{E_{0}+\varepsilon} .
$$

Substituting $\Sigma$ into Eq. (28) (note that $R=\Sigma$ and $T=1+\Sigma$ ) leads directly to Eq. (29):

$$
\frac{d \Sigma}{d \ln \left(\omega / E_{0}\right)}=-\frac{\Sigma^{*}(1+\Sigma)\left(|1+\Sigma|^{2}+|\Sigma|^{2}\right)}{\left[(1+2 \Sigma)\left(1+2 \Sigma^{*}\right)^{2}\right]} \frac{1}{\pi v_{F}}\left(g^{1 \perp}+g^{1 \|}-g^{2}\right) .
$$

Then we can see that the self-energy $\Sigma$ is also a power-law function of energy scale $\omega / E_{0}$, and the asymptotic power-law exponent is the same to the CDW exponent. Because in Eq. (18) the Green function of the lead will not be affected by scaling, Eq. (29) actually describes the scaling of tunneling potential $V$. If the line-width $\Gamma$ is assumed to have the same behavior as the self-energy $\Sigma$, then the device behavior can be obtained by using the Landauer-Buttiker formula

$$
j=\frac{i e}{\hbar} \int \frac{d \varepsilon}{2 \pi}\left[f_{L}(\varepsilon)-f_{R}(\varepsilon)\right] T(\varepsilon)
$$

where

$$
T(\varepsilon)=\operatorname{Tr}\left\{\frac{\Gamma_{L}(\varepsilon) \Gamma_{R}(\varepsilon)}{\Gamma_{L}(\varepsilon)+\Gamma_{R}(\varepsilon)}\left[G^{r}(\varepsilon)-G^{a}(\varepsilon)\right]\right\} .
$$

Here $j$ denotes the current of the device $f_{L}$, and $f_{R}$ are the Fermi-distribution functions of left and right leads, respectively. In Eq. (30b), $\operatorname{Tr}\{\}$ means taking the trace of the argument, and $G^{r}$ and $G^{a}$ are retarded and advanced Green functions, respectively. The transmission coefficient $T$ and therefore the differential conductance is proportional to the linewidth $\Gamma$, so the differential resistance has the exponent given by $\frac{1}{\pi v_{F}}\left(g^{1 \perp}+g^{1 \|}-g^{2}\right)$, the same as that for the CDW order.
In the case of $(5,0) \mathrm{CNT}$ thin array comprising $7 \mathrm{CNTs}$, we have used the values of $\widetilde{g}_{1}^{1 \perp}, \widetilde{g}_{1}^{2}, \widetilde{g}_{1}^{1 \|}$ and $\widetilde{g}_{4}^{1 \perp}, \widetilde{g}_{4}^{2}, \widetilde{g}_{4}^{1 \|}$ for the CDW order and numerically integrated the scaling equation to obtain the differential resistance in the two channels 1 and 4. Results are shown in Fig. 7. It is interesting to note that in the $\widetilde{g}_{1}^{1 \|, 1 \perp, 2}$ channel there is a minimum in differential resistance curve next to central peak. It can be seen that the most prominent feature of the CDW order is the peak in differential resistance around the Fermi level.

\section{RELEVANCE TO THE OBSERVED PHENOMENA}

\section{A. Description of the measured data}

An example of the measured differential resistance data is shown in Fig. 8. It is seen that at low temperatures there is a smooth dip that is characteristic of a fluctuating supercurrent in the system. In the inset we show the temperature dependence of the measured resistance. Its behavior informs of the system being in the $1 \mathrm{D}$ superconducting state. However, as the temperature was increased above $2.5 \mathrm{~K}$, a peak clearly emerged. The differential resistance was measured in the four-probe configuration and hence must reflect the intrinsic property of the system. This particular sample was prepared in a similar manner as described in Ref. 2, with the $(5,0)$ 


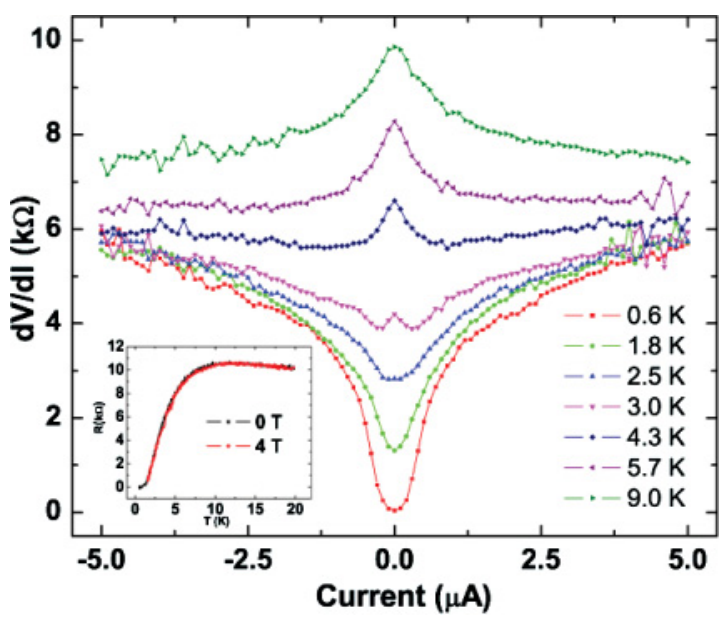

FIG. 8. (Color online) Differential resistance plotted as a function of the bias current. At temperatures above $2.5 \mathrm{~K}$, a zero-bias resistance peak is clearly seen. At low temperatures, a dip in resistance appears that indicates the existence of supercurrent. Inset is the corresponding $\mathrm{R}$-T curve under 0 and $4 \mathrm{~T}$ magnetic field. No difference is seen. The temperature dependence of the resistance and its magnetic field independence clearly demonstrate that the overall behavior is that of 1D superconductivity.

CNTs being recognized by their radial breathing mode (RBM) Raman shift of $550 \mathrm{~cm}^{-1}$. The fact that the measured results are insensitive to applied magnetic field, up to $9 \mathrm{~T}$ in addition to the smooth variation of resistance with both applied current and temperature, serves as evidence for the particular behaviors to arise from very thin arrays of the $(5,0)$ nanotubes to which the superconducting characteristics are attributed. This is in contrast to the magnetic field dependent behavior observed in the BKT-like transition. ${ }^{2-4}$ A plausible scenario is that the thin array(s) acts as the main link in the conducting path connecting the two voltage electrodes for this particular sample.

\section{B. A potential explanation based on the scaling results}

From the previous section it is seen that while the SS response is the most dominant for a thin array of $(5,0)$, the
CDW order can be close by in terms of energy since in the single $(5,0)$ CNT case it is the dominant order. Hence our interpretation is that the behavior shown in Fig. 8, especially the appearance of the differential resistance peak, can be due to the thermally activated higher-energy CDW order with the differential resistance peak around the Fermi level, as seen in the previous section. Such an explanation is plausible since the peak in differential resistance appears gradually with increasing temperature, centered at zero-bias current, while the overall temperature dependence of resistance is still given by that of 1D superconductivity (see inset to Fig. 8).

In accordance with the previous interpretation, there should be a continuous transformation of the 1D superconducting behavior (with a Peierls/CDW excited state) from that of a thin array of $(5,0)$ CNTs to the BKT-like transition in the transverse plane perpendicular to the $c$-axis of the aligned CNTs of a large array. ${ }^{2}$ Such a transformation may be characterized by an increasing energy separation (of the Peierls/CDW excited state from the superconducting ground state) as the array size increases, so that the characteristics of the Peierls/CDW order become more suppressed (with increasing array size) until they no longer appear in the relevant temperature regime; this occurs simultaneously with the appearance of the BKT-like transition. This can explain the absence of the differential resistance peak, e.g., in sample 1 of Ref. 2.

\section{CONCLUDING REMARKS}

In conclusion we have performed second-order RG calculations and obtained the fixed points in the strong coupling limit. Our results indicate the possibility of the Peierls/CDW order existing as a higher energy excited state in thin arrays of $(5,0)$ CNTs, with the 1D superconductivity being the ground state. Our interpretation provides a plausible explanation for the experimental observations. First-principles calculations along this direction are currently being pursued.

\section{ACKNOWLEDGMENT}

This work is supported by RGC grant HKUST9/CRF/08. *sheng@ust.hk

${ }^{1}$ Z. K. Tang, L. Zhang, N. Wang, X. X. Zhang, G. H. Wen, G. D. Li, J. N. Wang, C. T. Chan, and P. Sheng, Science 292, 2462 (2001).

${ }^{2}$ Z. Wang, W. Shi, H. Xie, T. Zhang, N. Wang, Z. K. Tang, X. X. Zhang, R. Lortz, P. Sheng, I. Sheikin, and A. Demuer, Phys. Rev. B 81, 174530 (2010).

${ }^{3}$ Z. Wang, W. Shi, H. Xie, T. Zhang, N. Wang, Z. Tang, X. Zhang, R. Lortz, P. Sheng, I. Sheikin, and A. Demuer, Physica Status Solidi B 247, 2968 (2010).

${ }^{4}$ R. Lortz, Q. C. Zhang, W. Shi, J. T. Ye, C. Y. Qiu, Z. Wang, H. T. He, P. Sheng, T. Z. Qian, Z. K. Tang, X. X. Zhang, J. N. Wang, and C. T. Chan, Proc. Nat. Acad. Sci. 106, 7299 (2009).

${ }^{5}$ C. Ieong, Z. Wang, W. Shi, Y. Wang, N. Wang, Z. K. Tang, P. Sheng, and R. Lortz, Phys. Rev. B, in print (arXiv:1103.1459).
${ }^{6}$ Z. M. Li, Z. K. Tang, G. G. Siu, and I. Bozovic, Appl. Phys. Lett. 84, 4101 (2004).

${ }^{7}$ Z. M. Li, H. J. Liu, J. T. Ye, C. T. Chan, and Z. K. Tang, Appl. Phys. A 78, 1121 (2004).

${ }^{8}$ Z. K. Tang, H. D. Sun, J. Wang, J. Chen, and G. Li, Appl. Phys. Lett. 73, 2287 (1998).

${ }^{9}$ I. L. Li, G. D. Li, H. J. Liu, C. T. Chan, and Z. K. Tang, Appl. Phys. Lett. 82, 1467 (2003).

${ }^{10}$ H. J. Liu and C. T. Chan, Phys. Rev. B 66, 115416 (2002).

${ }^{11}$ L. X. Benedict, V. H. Crespi, S. G. Louie, and M. L. Cohen, Phys. Rev. B 52, 14935 (1995).

${ }^{12}$ Y. A. Krotov, D. H. Lee, and S. G. Louie, Phys. Rev. Lett. 78, 4245 (1997).

${ }^{13}$ J. Gonzalez, Phys. Rev. Lett. 88, 076403 (2002). 
${ }^{14}$ M. Kociak, A. Y. Kasumov, S. Gueron, B. Reulet, I. I. Khodos, Y. B. Gorbatov, V. T. Volkov, L. Vaccarini, and H. Bouchiat, Phys. Rev. Lett. 86, 2416 (2001).

${ }^{15}$ J. Gonzalez, Phys. Rev. B 67, 014528 (2003).

${ }^{16}$ A. D. Martino and R. Egger, Phys. Rev. B 70, 014508 (2004).

${ }^{17}$ J. Gonzalez and J. V. Alvarez, Phys. Rev. B 70, 045410 (2004)

${ }^{18}$ I. Takesue, J. Haruyama, N. Kobayashi, S. Chiashi, S. Maruyama, T. Sugai, and H. Shinohara, Phys. Rev. Lett. 96, 057001 (2006).

${ }^{19}$ N. Murata, J. Haruyama, Y. Ueda, M. Matsudaira, H. Karino, Y. Yagi, E. Einarsson, S. Chiashi, S. Maruyama, T. Sugai, N. Kishi, and H. Shinohara, Phys. Rev. B 76, 245424 (2007).

${ }^{20}$ T. Tsuneta, L. Lechner, and P. J. Hakonen, Phys. Rev. Lett. 98, 087002 (2007).

${ }^{21}$ Y. B. Gaididei and V. M. Loktev, Low Temp. Phys. 32, 1111 (2006).

${ }^{22}$ E. Perfetto and J. Gonzalez, Phys. Rev. B 74, 201403(R) (2006).

${ }^{23}$ J. Noffsinger and M. L. Cohen, Phys. Rev. B 83, 165420 (2011).

${ }^{24}$ N. Murata, J. Haruyama, J. Reppert, A. M. Rao, T. Koretsune, S. Saito, M. Matsudaira, and Y. Yagi, Phys. Rev. Lett. 101, 027002 (2008).

${ }^{25}$ J. Haruyama, M. Matsudaira, J. Reppert, A. Rao, T. Koretsune, S. Saito, H. Sano, and Y. Iye, J. Supercond. Nov. Magn. 24, 111 (2011).

${ }^{26}$ A. Sedeki, L. G. Caron, and C. Bourbonnais, Phys. Rev. B 65, 140515(R) (2002).

${ }^{27}$ K. P. Bohnen, R. Heid, and C. T. Chan, J. Phys. Condens. Matter. 21, 084206 (2009).

${ }^{28}$ K. P. Bohnen, R. Heid, H. J. Liu, and C. T. Chan, Phys. Rev. Lett. 93, 245501 (2004).

${ }^{29}$ D. Connetable, G. M. Rignanese, J. C. Charlier, and X. Blase, Phys. Rev. Lett. 94, 015503 (2005).

${ }^{30}$ P. M. Singer, P. Wzietek, H. Alloul, F. Simon, and H. Kuzmany, Phys. Rev. Lett. 95, 236403 (2005).

${ }^{31}$ B. Dora, M. Gulacsi, F. Simon, and H. Kuzmany, Phys. Rev. Lett. 99, 166402 (2007).

${ }^{32}$ M. V. Fernandez-Serra and X. Blase, Phys. Rev. B 77, 195115 (2008).

${ }^{33}$ L. Balents and M. P. A. Fisher, Phys. Rev. B 55, 11973 (1997).

${ }^{34}$ K. Kamide, M. Nishida, and S. Kurihara, Physica. E 18, 218 (2003).

${ }^{35}$ K. Kamide, T. Kimura, M. Nishida, and S. Kurihara, Phys. Rev. B 68, 024506 (2003).

${ }^{36}$ R. Barnett, E. Demler, and E. Kaxiras, Phys. Rev. B 71, 035429 (2005).

${ }^{37}$ R. Barnett, E. Demler, and E. Kaxiras, Solid State Commun. 135, 335 (2005).
${ }^{38}$ K. Iyakutti, A. Bodapati, X. H. Peng, P. Keblinski, and S. K. Nayak, Phys. Rev. B 73, 035413 (2006).

${ }^{39}$ S. Bellucci, M. Cini, P. Onorato, and E. Perfetto, J. Phys. Condens. Matter. 18, S2115 (2006).

${ }^{40}$ S. Bellucci, M. Cini, P. Onorato, and E. Perfetto, Phys. Rev. B 75, 014523 (2007).

${ }^{41}$ V. L. Berezinskii, Sov. Phys. JETP 32, 493 (1971).

${ }^{42}$ V. L. Berezinskii, Sov. Phys. JETP 34, 610 (1972).

${ }^{43}$ J. M. Kosterlitz and D. J. Thouless, J. Phys. C 6, 1181 (1973).

${ }^{44}$ S. Tomonaga, Prog. Theor. Phys. 5, 544 (1950).

${ }^{45}$ J. M. Luttinger, J. Math. Phys. 4, 1154 (1963).

${ }^{46}$ D. C. Mattis and E. H. Lieb, J. Math. Phys. 6, 304 (1965).

${ }^{47}$ J. D. Delft and H. Schoeller, Ann. Phys. (Leipzig) 7, 225 (1998).

${ }^{48}$ A. O. Gogolin, A. A. Nersesyan, and A. M. Tsvelik, Bosonization and Strongly Correlated Systems (Cambridge University Press Cambridge, UK, 1998).

${ }^{49}$ J. Voit, Rep. Prog. Phys. 57, 977 (1994).

${ }^{50}$ M. Bockrath, D. H. Cobden, J. Lu, A. G. Rinzler, R. E. Smalley, L. Balents, and P. L. McEuen, Nature 397, 598 (1999).

${ }^{51}$ C. L. Kane and M. P. A. Fisher, Phys. Rev. B 4615233 (1992).

${ }^{52}$ J. Solyom, Adv. in Phys. 28, 201 (1979).

${ }^{53}$ N. Menyhard and J. Solyom, J. Low Temp. Phys. 12, 529 (1973).

${ }^{54}$ R. Shankar, Rev. Mod. Phys. 66, 129 (1994).

${ }^{55}$ C. M. Varma, and A. Zawadowski, Phys. Rev. B 32, 7399 (1985).

${ }^{56}$ K. Penc and J. Solyom, Phys. Rev. B 41, 704 (1990).

${ }^{57}$ J. Solyom, J. Low Temp. Phys. 12, 547 (1973).

${ }^{58}$ T. Giamarchi, Quantum Physics in One Dimension (DPMC, University of Geneva, 2003).

${ }^{59}$ J. Gonzalez and E. Perfetto, Phys. Rev. B 72, 205406 (2006).

${ }^{60}$ K. Kamide, T. Kimura, M. Nishida, and S. Kurihara, Phys. Rev. B 68, 024506 (2003).

${ }^{61}$ D. Carpentier and E. Orignac, Phys. Rev. B 74, 085409 (2006).

${ }^{62}$ D. W. Wang, A. J. Millis, and S. Das Sarma, Phys. Rev. B 64, 193307 (2001).

${ }^{63}$ K. Kajiwara, M. Tsuchiizu, Y. Suzumura, and C. Bourbonnais, J. Phys. Soc. Jpn. 78, 104702 (2009).

${ }^{64}$ K. A. Matveev, D. X. Yue, and L. I. Glazman, Phys. Rev. Lett. 71, 3351 (1993).

${ }^{65}$ D. X. Yue, L. I. Glazman, and K. A. Matveev, Phys. Rev. B 49, 1966 (1994).

${ }^{66}$ P. W. Anderson, J. Phys. C 3, 2436 (1970). 